\title{
Synthesis, Structures, and Properties of Neutral and Radical Cationic S,C,C-Bridged Triphenylamines
}

\author{
Shin-ichiro Kato, ${ }^{* \dagger}$ Takanori Matsuoka, ${ }^{\dagger}$ Shuichi Suzuki, ${ }^{\ddagger}$ Motoko S. Asano, ${ }^{\S}$ \\ Toshitada Yoshihara, ${ }^{\ddagger}$ Seiji Tobita, ${ }^{\ddagger}$ Taisuke Matsumoto, ${ }^{\mathbb{P}}$ Chitoshi Kitamura ${ }^{\dagger}$
}

$\dagger$ Department of Materials Science, School of Engineering, The University of Shiga Prefecture, 2500 Hassaka-cho, Hikone, Shiga 522-8533, Japan

$\$$ Graduate School of Engineering Science, Osaka University, 1-3 Machikaneyama, Toyonaka, Osaka 560-8531, Japan

$\S$ Division of Molecular Science, Faculty of Science and Technology, Gunma University, 1-5-1

Tenjin-cho, Kiryu, Gunma 376-8515, Japan

PInstitute for Materials Chemistry and Engineering (IMCE), Kyushu University, 6-1 Kasuga-koh-en, Kasuga 816-8580, Japan

Table of Contents

1. General experimental methods (S2)

2. Synthesis (S2)

3. X-ray crystallographic data (S6)

4. Photophysical data (S9)

5. Electrochemical data (S10)

6. Theoretical data (S11)

7. ${ }^{1} \mathrm{H}$ and ${ }^{13} \mathrm{C}$ NMR spectral data (S20)

8. ESR spectral data (S25)

9. References (S26) 


\section{General experimental methods}

Commercially available reagents and solvents were used as received; $(n-\mathrm{Bu})_{2} \mathrm{O}$ was dried with $3 \mathrm{~A}$ molecular sieves prior to use. Oil bath was used as the heat source. Column chromatography and plug filtrations were carried out with $\mathrm{SiO}_{2}$ or alumina. Thin-layer chromatography (TLC) was conducted on aluminum sheets coated with $\mathrm{SiO}_{2} 60 \mathrm{~F}_{254}$. Melting points (M.p.) were measured with a hot-stage apparatus and are uncorrected. Recycling gel-permeation chromatography was performed with UV detectors using $1 \mathrm{H}$ and $2 \mathrm{H}$ polystyrene columns eluting with $\mathrm{CHCl}_{3} .{ }^{1} \mathrm{H}$ and ${ }^{13} \mathrm{C} \mathrm{NMR}$ spectra were recorded on a spectrometer at $400 \mathrm{MHz}$ for ${ }^{1} \mathrm{H}$ and $100 \mathrm{MHz}$ for ${ }^{13} \mathrm{C}$. ESI-MS spectrometries were conducted in a positive or negative mode. Electronic absorption and fluorescence spectra were measured in a cuvette of $1 \mathrm{~cm}$ at room temperature. Absolute fluorescence quantum yields were determined by an integrating sphere system. Cyclic voltammetry was performed by using a cell equipped with a platinum as working electrode, a platinum wire as counter electrodes, and $\mathrm{Ag} / \mathrm{AgNO}$ as a referential electrode. All electrochemical measurements were performed in $\mathrm{CH}_{2} \mathrm{Cl}_{2}$ solution (ca. $\left.5 \times 10^{-4} \mathrm{~mol} \mathrm{~L}^{-1}\right)$ containing $0.1 \mathrm{~mol} \mathrm{~L}^{-1}(n-\mathrm{Bu})_{4} \mathrm{NPF}_{6}$ at room temperature. All potentials are referenced to the ferrocenium/ferrocene $\left(\mathrm{Fc}^{+} / \mathrm{Fc}\right)$ couple, used as a standard. X-ray diffraction analyses for a single crystal were performed with an X-ray diffractometer for which a Mo-K $\alpha$ was used. Quantum chemical calculations were performed by Gaussian 09 package of ab initio MO calculations. ${ }^{1}$ ESR spectrum was recorded on a spectrometer equipped with a microwave frequency counter and a magnetic fieldmeter. ESR signals were transferred to a PC through a digital multimeter and a GPIB interface. For the spectrum in Figure 6, X band microwave was used (microwave frequency $=9.229$ $\mathrm{GHz}$ ). Sample was dissolved in super dehydrated dichlomethane, and the solution was sealed in a quartz tube ( $3 \mathrm{~mm}$ o.d.) under nitrogen atmosphere. ESR spectrum simulation was performed by using EasySpin. ${ }^{2}$

\section{Synthesis}

\section{Preparation of iodide 6}

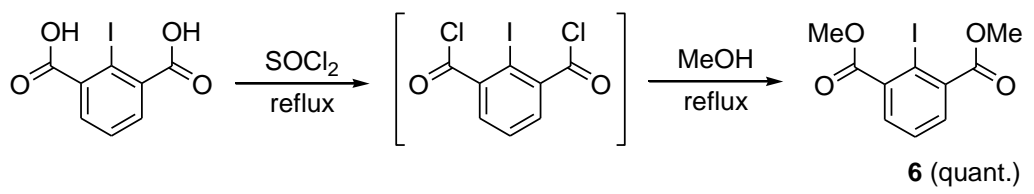

A mixture of 2-iodoisophthalic acid $(5.26 \mathrm{~g}, 18.0 \mathrm{mmol})$ and $\mathrm{SOCl}_{2}(40 \mathrm{~mL})$ was refluxed under nitrogen atmosphere for $24 \mathrm{~h}$. Removal of the excess $\mathrm{SOCl}_{2}$ under reduced pressure afforded 2iodobenzene-1,3-dicarbonyl dichloride as white solids. A solution of the obtained acyl chloride in dry methanol $(200 \mathrm{~mL})$ was refluxed under nitrogen atmosphere for $15 \mathrm{~h}$. Removal of methanol under reduced pressure afforded iodide 6 (5.72 g, $17.9 \mathrm{mmol}$, quant.) as pale brown oil. ${ }^{1} \mathrm{H}$ NMR (400 MHz, $\left.\mathrm{CDCl}_{3}\right): \delta 3.96(6 \mathrm{H}, \mathrm{s}), 7.45(1 \mathrm{H}, \mathrm{t}, J=7.7 \mathrm{~Hz}), 7.63(2 \mathrm{H}, \mathrm{d}, J=7.7 \mathrm{~Hz}) \mathrm{ppm}$. The ${ }^{1} \mathrm{H}$ NMR data are agreement with those in the literature. ${ }^{3}$ 
Preparation of methyl ester 7

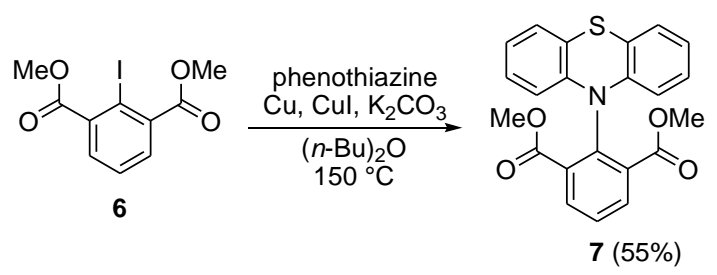

A suspension of iodide $6(1.12 \mathrm{~g}, 3.50 \mathrm{mmol})$, phenothiazine $(0.536 \mathrm{~g}, 2.69 \mathrm{mmol}), \mathrm{K}_{2} \mathrm{CO}_{3}(0.836 \mathrm{~g}$, $6.05 \mathrm{mmol}), \mathrm{Cu}(77 \mathrm{mg}, 1.21 \mathrm{mmol})$, and $\mathrm{CuI}(56 \mathrm{mg}, 0.296 \mathrm{mmol})$ in $(n-\mathrm{Bu})_{2} \mathrm{O}(20 \mathrm{~mL})$ was bubbled with nitrogen for $15 \mathrm{~min}$ and refluxed $\left(150^{\circ} \mathrm{C}\right)$ under nitrogen atmosphere for $48 \mathrm{~h}$. After the resulting mixture was diluted with toluene, insoluble material was removed by filtration through a bed of Celite, and the filtrate was evaporated under reduced pressure. The resulting solid material was washed with hexane and collected by filtration. The obtained material was purified by column chromatography $\left(\mathrm{SiO}_{2}\right.$, toluene; AcOEt/toluene 1:20) and recrystallization from EtOH/hexane to afford methyl ester 7 (0.576 g, $1.47 \mathrm{mmol}, 55 \%)$ as pale yellow solids. M.p. $171-172{ }^{\circ} \mathrm{C} ;{ }^{1} \mathrm{H} \mathrm{NMR}\left(\mathrm{CDCl}_{3}, 400 \mathrm{MHz}\right) \delta$ $8.22(2 \mathrm{H}, \mathrm{d}, J=7.8 \mathrm{~Hz}), 7.67(1 \mathrm{H}, \mathrm{t}, J=7.8 \mathrm{~Hz}), 6.91(2 \mathrm{H}, \mathrm{dd}, J=1.8 \& 7.1 \mathrm{~Hz}), 6.76(2 \mathrm{H}, \mathrm{td}, J=1.8$ \& $7.1 \mathrm{~Hz}), 6.72(2 \mathrm{H}, \mathrm{td}, J=1.8 \& 7.1 \mathrm{~Hz}), 5.85(2 \mathrm{H}, \mathrm{dd}, J=1.8 \& 7.1 \mathrm{~Hz}), 3.74(6 \mathrm{H}, \mathrm{s}) \mathrm{ppm} ;{ }^{13} \mathrm{C}$ $\mathrm{NMR}\left(\mathrm{CDCl}_{3}, 100 \mathrm{MHz}\right) \delta 166.0,142.9,138.8,135.7,134.7,129.0,126.8,126.4,122.3,118.9,115.1$, 53.0 ppm (12 signals out of 12 expected); UV-vis $\left(\mathrm{CH}_{2} \mathrm{Cl}_{2}\right): \lambda_{\max }{ }^{\text {abs }}(\varepsilon) 320$ (4000), 253 (27000) nm; HR-FT-MS (positive): $\mathrm{m} / z$ calcd for $\mathrm{C}_{22} \mathrm{H}_{18} \mathrm{NO}_{4} \mathrm{~S}^{+} 392.0957$, found $392.0942\left[(\mathrm{M}+\mathrm{H})^{+}\right]$.

\section{Preparation of alcohol 8}

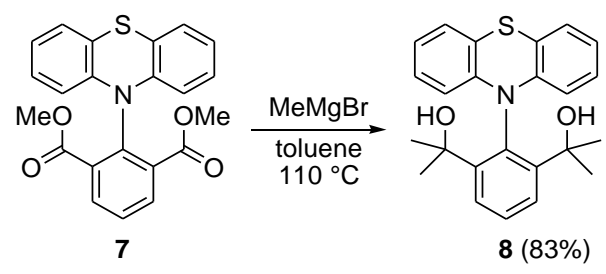

To a solution of methyl ester $7(3.00 \mathrm{~g}, 7.67 \mathrm{mmol})$ in toluene $(120 \mathrm{~mL})$ was added dropwise methylmagnesium bromide THF solution ( $\left.3 \mathrm{~mol} \mathrm{~L}^{-1}, 40 \mathrm{~mL}, 120 \mathrm{mmol}\right)$, and the solution was refluxed $\left(110{ }^{\circ} \mathrm{C}\right)$ under nitrogen atmosphere for $15 \mathrm{~h}$. After addition of water, the organic layer was separated, and the aqueous phase was extracted with toluene/AcOEt $(1: 1, \mathrm{v} / \mathrm{v})$. The combined organic layer was washed with saturated aqueous $\mathrm{NaCl}$, dried over $\mathrm{Na}_{2} \mathrm{SO}_{4}$, and evaporated under reduced pressure. The residue was purified by column chromatography $\left(\mathrm{SiO}_{2}, \mathrm{AcOEt} /\right.$ toluene 1:5) to afford alcohol 8 (2.48 g, $6.34 \mathrm{mmol}, 83 \%$ ) as yellow solids. M.p. $154-156{ }^{\circ} \mathrm{C} .{ }^{1} \mathrm{H}$ NMR (400 MHz, DMSO- $d_{6}$, two atrop isomers): $\delta 8.33,8.01,7.84(2 \mathrm{H}, 3 \mathrm{~d}, J=7.7 \mathrm{~Hz}), 7.70,7.47(1 \mathrm{H}, 2 \mathrm{t}, J=7.7 \mathrm{~Hz}), 6.85-6.64(6 \mathrm{H}, \mathrm{m})$, 5.76, $5.73(2 \mathrm{H}, 2 \mathrm{~d}, J=8.4 \mathrm{~Hz}), 5.25,4.70(2 \mathrm{H}, 2 \mathrm{~s}), 2.41,1.40(12 \mathrm{H}, 2 \mathrm{~s}) \mathrm{ppm} ;{ }^{13} \mathrm{C}$ NMR (100 MHz, DMSO- $\left.d_{6}\right): \delta 198.6,149.9,149.00,148.98,144.9,144.2,139.9,133.4,132.8,132.02,132.01,130.1$, 129.2, 129.0, 128.3, 126.8, 126.3, 126.1, 125.6, 122.2, 121.4, 117.21, 117.19, 116.5, 115.73, 115.69, $72.41,72.39,71.3,32.5,30.9,30.1 \mathrm{ppm}$ (32 signals out of 32 expected); $U V$-vis $\left(\mathrm{CH}_{2} \mathrm{Cl}_{2}\right): \lambda_{\max }{ }^{\text {abs }}(\varepsilon)$ 
320 (4300), 258 (56200) nm; HR-FT-MS (positive): $m / z$ calcd for $\mathrm{C}_{24} \mathrm{H}_{26} \mathrm{NO}_{2} \mathrm{~S}^{+} 392.1684$, found $392.1671\left[(\mathrm{M}+\mathrm{H})^{+}\right]$.

\section{Preparation of bridged N-phenylphenothiazines 4 and 5}

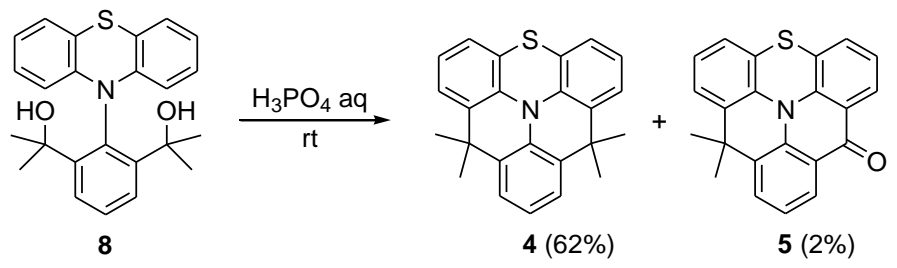

A suspension of $8(1.65 \mathrm{~g}, 4.21 \mathrm{mmol})$ in $\mathrm{H}_{3} \mathrm{PO}_{4}(85 \mathrm{wt} \%, 20 \mathrm{~mL})$ was stirred at room temperature for $4 \mathrm{~h}$. After addition of aqueous $\mathrm{NaOH}\left(1 \mathrm{~mol} \mathrm{~L} \mathrm{~L}^{-1}, 300 \mathrm{~mL}\right)$, the mixture was extracted with toluene/AcOEt $(1: 1, \mathrm{v} / \mathrm{v})$, and the resulting organic layer was dried over $\mathrm{Na}_{2} \mathrm{SO}_{4}$ and evaporated under reduced pressure. The residue was purified by column chromatography $\left(\mathrm{Al}_{2} \mathrm{O}_{3}\right.$, hexane/toluene 2:1; toluene; toluene/AcOEt 9:1) to give 4 (1.02 g, $2.87 \mathrm{mmol}, 62 \%)$ as pale yellow solids and 5 (36 $\mathrm{mg}$, $0.10 \mathrm{mmol}, 2 \%)$ as yellow solids. An analytically pure sample of 5 was obtained by recycling GPC eluting with $\mathrm{CHCl}_{3}$. 4: M.p. $194-196{ }^{\circ} \mathrm{C}$; ${ }^{1} \mathrm{H} \mathrm{NMR}\left(\mathrm{CDCl}_{3} / \mathrm{Et}_{3} \mathrm{~N}, 400 \mathrm{MHz}\right) \delta 7.32(2 \mathrm{H}, \mathrm{d}, J=7.6 \mathrm{~Hz})$, $7.15(2 \mathrm{H}, \mathrm{dd}, J=1.6 \& 7.3 \mathrm{~Hz}), 7.11(1 \mathrm{H}, \mathrm{t}, J=7.6 \mathrm{~Hz}), 6.90(2 \mathrm{H}, \mathrm{t}, J=6.6 \mathrm{~Hz}), 6.77(2 \mathrm{H}, \mathrm{dd}, J=1.6$ \& $7.3 \mathrm{~Hz}), 1.65(12 \mathrm{H}, \mathrm{s}) \mathrm{ppm} ;{ }^{13} \mathrm{C} \mathrm{NMR}\left(\mathrm{CDCl}_{3} / \mathrm{Et}_{3} \mathrm{~N}, 100 \mathrm{MHz}\right) \delta 134.3,131.9,131.0,129.7,124.34$, 124.27, 124.23, 123.49, 123.36, 116.4, 35.5, 32.9 (12 signals out of 12 expected); UV-vis $\left(\mathrm{CH}_{2} \mathrm{Cl}_{2}\right)$; $\lambda_{\max }{ }^{\text {abs }}(\varepsilon) 280$ (42600), 303 (15900), 334 (4900); HR-ESI-MS (positive): $m / z$ calcd for $\mathrm{C}_{24} \mathrm{H}_{22} \mathrm{NS}^{+}$ 356.1473, found 356.1461 [(M+H $)^{+}$]. 5: M.p. $166-168{ }^{\circ} \mathrm{C} ;{ }^{1} \mathrm{H} \mathrm{NMR}\left(\mathrm{CDCl}_{3}, 400 \mathrm{MHz}\right) \delta 8.49(1 \mathrm{H}$, $\mathrm{dd}, J=1.8 \& 7.6 \mathrm{~Hz}), 8.23(1 \mathrm{H}, \mathrm{dd}, J=1.8 \& 7.6 \mathrm{~Hz}), 7.85(1 \mathrm{H}, \mathrm{dd}, J=1.8 \& 7.6 \mathrm{~Hz}), 7.42(1 \mathrm{H}, \mathrm{t}, J$ $=7.6 \mathrm{~Hz}), 7.29(1 \mathrm{H}, \mathrm{dd}, J=1.8 \& 7.6 \mathrm{~Hz}), 7.25(1 \mathrm{H}, \mathrm{dd}, J=1.8 \& 7.8 \mathrm{~Hz}), 7.18(1 \mathrm{H}, \mathrm{t}, J=7.6 \mathrm{~Hz})$, $7.07(1 \mathrm{H}, \mathrm{t}, J=7.6 \mathrm{~Hz}), 6.88(1 \mathrm{H}, \mathrm{dd}, J=1.8,7.6 \mathrm{~Hz}), 1.67(6 \mathrm{H}, \mathrm{s}) \mathrm{ppm} ;{ }^{13} \mathrm{C} \mathrm{NMR}\left(\mathrm{CDCl}_{3}, 100 \mathrm{MHz}\right)$ $\delta 177.1,137.3,135.2,133.5,132.3,131.2,129.7,126.6,126.2,125.57,125.48,125.28,124.5,124.0$, $122.6,121.7,119.5,118.5,35.9,34.2 \mathrm{ppm}$ (21 signals out of 21 expected); $\mathrm{UV}-\mathrm{vis}\left(\mathrm{CH}_{2} \mathrm{Cl}_{2}\right): \lambda_{\max }{ }^{\text {abs }}$ (ع) 261 (42600), 327 (4600), 447 (8400); HR-ESI-MS (positive): $\mathrm{m} / z$ calcd for $\mathrm{C}_{22} \mathrm{H}_{15} \mathrm{NOS}^{+} 342.0953$, found $342.0940\left[(\mathrm{M}+\mathrm{H})^{+}\right]$. 

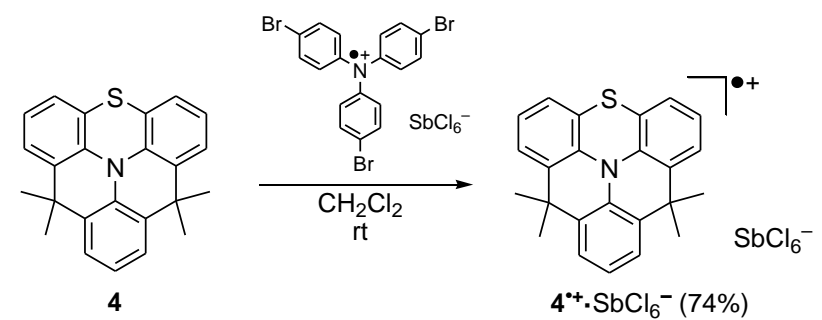

To a solution of $4(30 \mathrm{mg}, 84 \mu \mathrm{mol})$ in $\mathrm{CH}_{2} \mathrm{Cl}_{2}$ was added tris(4-bromophenyl)ammoniumyl hexachloroantimonate $(70 \mathrm{mg}, 84 \mu \mathrm{mol})$, and the mixture was stirred at room temperature for $1 \mathrm{~h}$ under nitrogen atmosphere. After the mixture was evaporated, the residue was washed with $\mathrm{Et}_{2} \mathrm{O}$. The insoluble material was collected by filtration to give $\mathbf{4}^{\cdot+} \cdot \mathbf{S b C l}^{-}(43 \mathrm{mg}, 62 \mu \mathrm{mol}, 74 \%)$ as black solids. M.p. $153-154{ }^{\circ} \mathrm{C}$ (decomp.); ${ }^{1} \mathrm{H}$ NMR $\left(\mathrm{CD}_{2} \mathrm{Cl}_{2}, 400 \mathrm{MHz}\right)$ No peak appeared; UV-vis $\left(\mathrm{CH}_{2} \mathrm{Cl}_{2}\right)$ : $\lambda_{\max }{ }^{\text {abs }}(\varepsilon) 298$ (33500), 514 (8000), 928 (3000), 1058 (4800) nm; HR-ESI-MS (positive): $\mathrm{m} / z$ calcd for $\mathrm{C}_{24} \mathrm{H}_{21} \mathrm{NS}^{+}$355.1389, found $355.1394\left[\left(\mathrm{M}-\mathrm{SbCl}_{6}\right)^{+}\right.$]; HR-ESI-MS (negative): $\mathrm{m} / z$ calcd for $\mathrm{SbCl}_{6}$ 330.7175 , found $330.7176\left[\left(\mathrm{M}-\mathrm{C}_{24} \mathrm{H}_{21} \mathrm{NS}\right)^{-}\right]$; elemental analysis calcd (\%) for $\mathrm{C}_{24} \mathrm{H}_{21} \mathrm{Cl}_{6} \mathrm{NSSb} \mathrm{C}$ 41.78, H 3.07, N 2.03; found: C 42.00, H 3.19, N 1.99 .

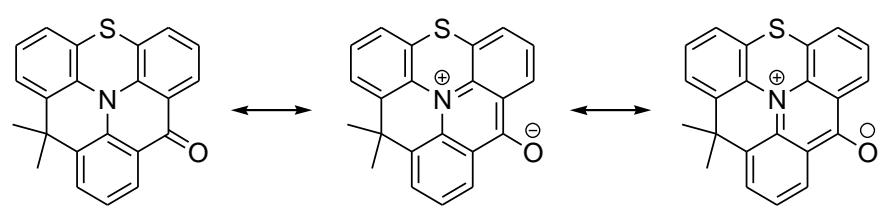

5

Scheme S1. Resonance structure of $\mathbf{5}$ proposed by its X-ray crystal structure. 


\section{X-ray crystallographic data}

Using Olex $2,{ }^{4}$ the structure was solved with the ShelXT ${ }^{5}$ structure solution program using Intrinsic Phasing and refined with the ShelXL ${ }^{6}$ refinement package using Least Squares minimization.

4 (CCDC 1949205): crystal data at $123 \mathrm{~K}, \mathrm{C}_{24} \mathrm{H}_{21} \mathrm{NS}, M_{\mathrm{r}}=355.48$, Monoclinic, space group $P 2_{1}, D_{\text {calcd }}$ $=1.337 \mathrm{~g} / \mathrm{cm}^{3}, Z=4, a=10.2194(3) \AA, b=10.0299(4) \AA, c=17.2309(6) \AA, \beta=90.155(3)^{\circ}, V=$ 1766.15(11) $\AA^{3}$; Mo- $K \alpha$ radiation, $\lambda=0.71073 \AA, \mu=0.190 \mathrm{~mm}^{-1}$. A pale yellow crystal was obtained from $\mathrm{CH}_{2} \mathrm{Cl}_{2} /$ hexane solution at $5{ }^{\circ} \mathrm{C}$. Numbers of measured and unique reflections were 13704 and 11709 , respectively $\left(R_{\text {sigma }}=0.0459\right)$. Final $R(F)=0.0410$ for 479 parameters and 11709 reflections with $I>2 \sigma(I)$ (for all data, $R(F)$ and $w R\left(F^{2}\right)$ values are 0.0496 and 0.1018 , respectively).

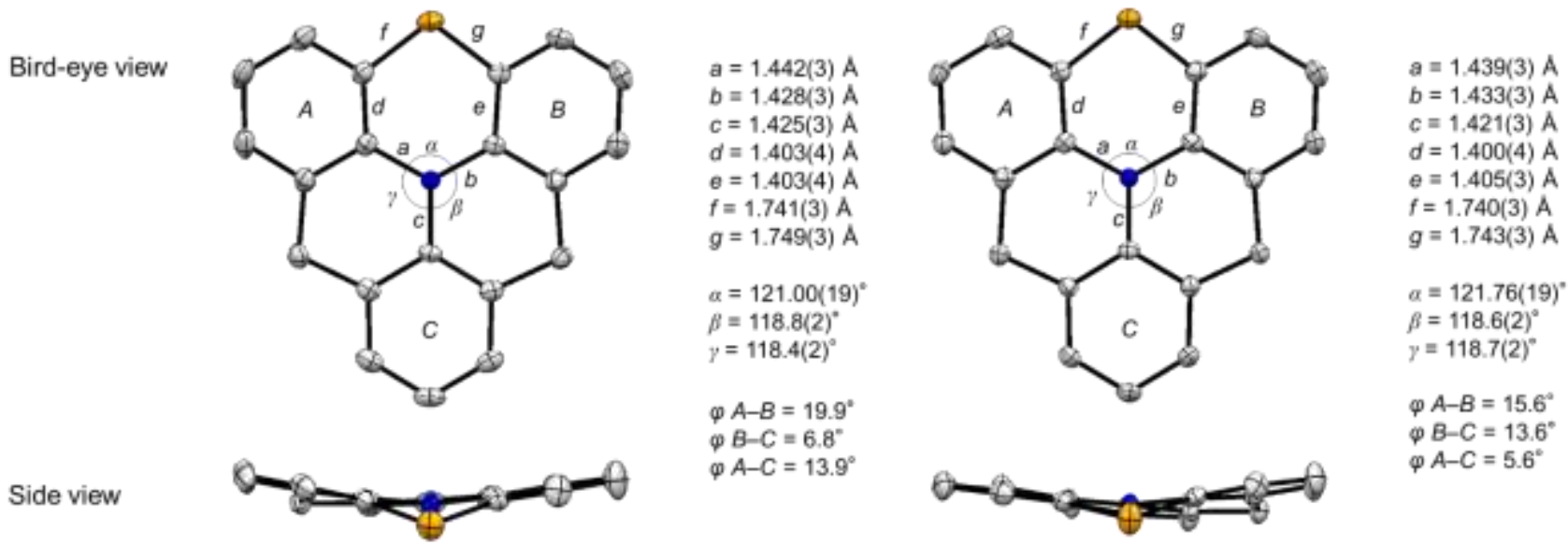

Figure S1. X-ray crystal structure of $\mathbf{4}$ from bird-eye view (top) and side view (bottom). Two independent molecules are in the unit cell. Displacement ellipsoids are shown at the 50\% probability level. Hydrogen atoms and Me groups are omitted for clarity.

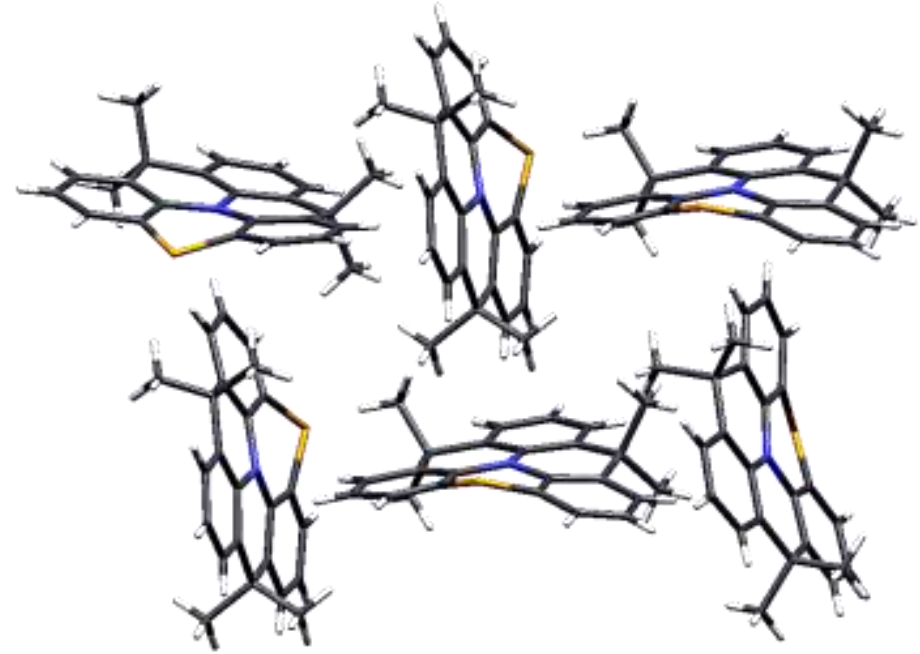

Figure S2. Arrangement of neighboring molecules of $\mathbf{4}$ in the crystal packing. 
5 (CCDC 1949206): Crystal data at $123 \mathrm{~K}$ for $\mathrm{C}_{22} \mathrm{H}_{15} \mathrm{NOS}, M_{\mathrm{r}}=341.41$, Triclinic, space group $P-1$, $D_{\text {calcd }}=1.501 \mathrm{~g} \mathrm{~cm}^{-3}, Z=2, a=9.1784(7) \AA, b=9.3524(6) \AA, c=10.5444(9) \AA, \alpha=66.322(7)^{\circ}, \beta=$ $67.937(7)^{\circ}, \gamma=72.242(6)^{\circ}, V=755.46(11) \AA^{3}$; Mo- $K \alpha$ radiation, $\lambda=0.71073 \AA, \mu=0.224 \mathrm{~mm}^{-1}$. A yellow crystal was obtained from $\mathrm{CH}_{2} \mathrm{Cl}_{2}$ /hexane solution at $25^{\circ} \mathrm{C}$. Numbers of measured and unique reflections were 11982 and 3464, respectively $\left(R_{\text {sigma }}=0.0392\right)$. Final $R(F)=0.0555$ for 228 parameters and 3464 reflections with $I>2 \sigma(I)$ (for all data, $R(F)$ and $w R\left(F^{2}\right)$ values are 0.0624 and 0.1632 , respectively).

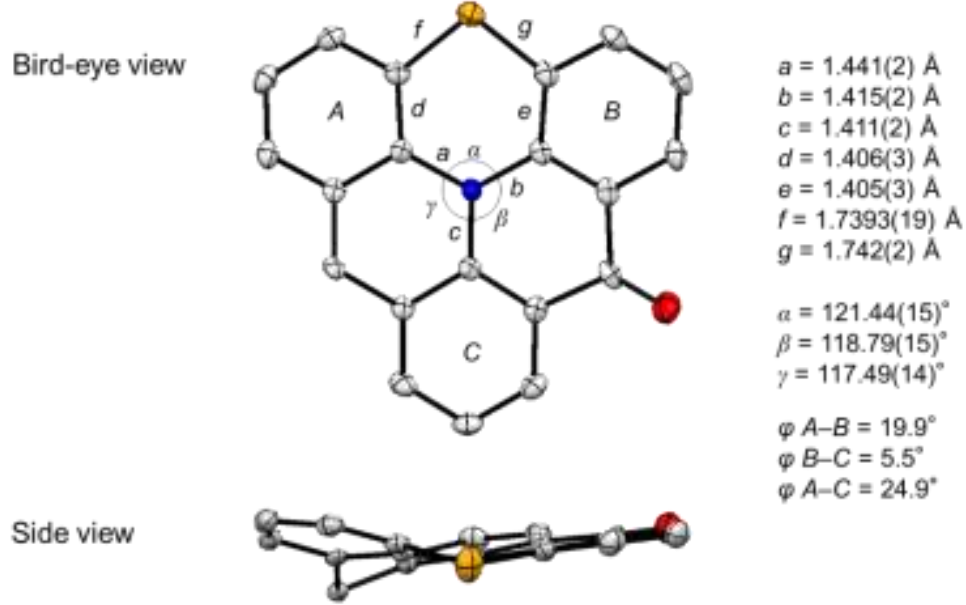

Figure S3. X-ray crystal structure of 5 from bird-eye view (top) and side view (bottom). Displacement ellipsoids are shown at the $50 \%$ probability level. Hydrogen atoms and Me groups are omitted for clarity.
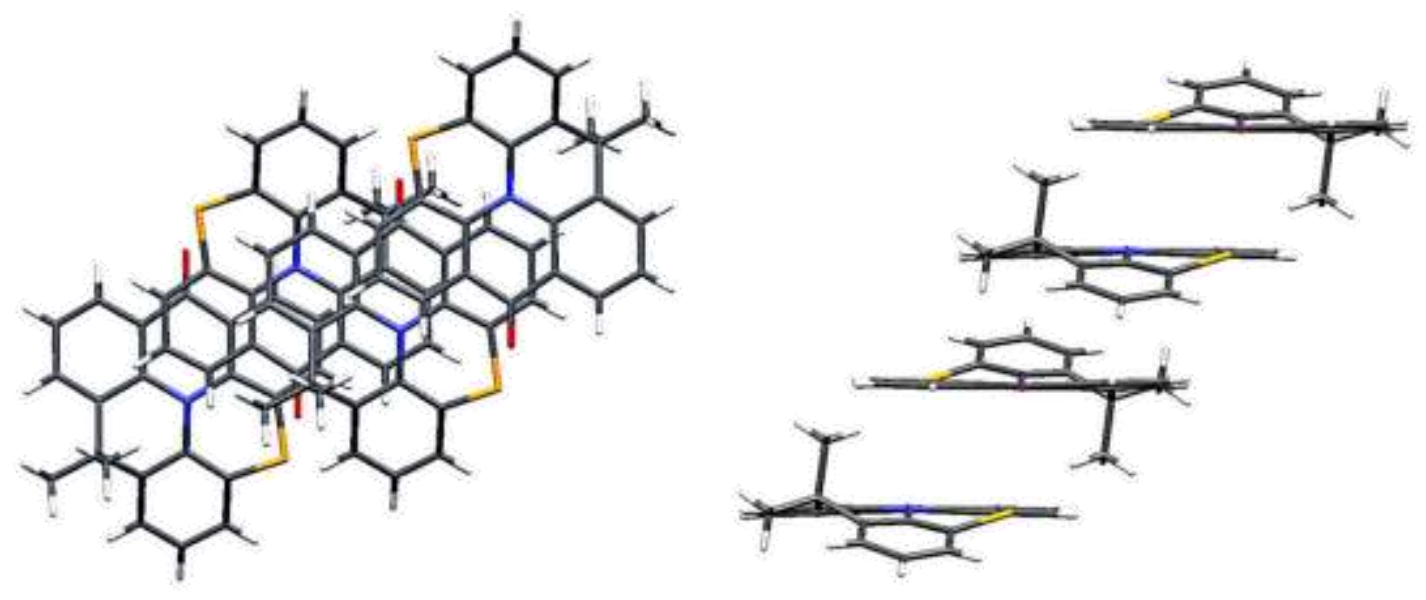

Figure S4. Arrangement of neighboring molecules of $\mathbf{5}$ in the crystal packing. 
$\mathbf{4}^{\cdot+} \cdot \mathbf{S b C l}^{-}$(CCDC 1949207): Crystal data at $123 \mathrm{~K}$ for $\mathrm{C}_{24} \mathrm{H}_{21} \mathrm{Cl}_{6} \mathrm{NSSb}, M_{\mathrm{r}}=689.93$, Monoclinic, space group $P 2{ }_{1} / c, D_{\text {calcd }}=1.826 \mathrm{~g} \mathrm{~cm}^{-3}, Z=4, a=9.7379(2) \AA, b=17.7260(4) \AA, c=14.9795(3) \AA$, $\beta=103.933(2)^{\circ}, V=2509.60(9) \AA^{3}$; Mo-Ka radiation, $\lambda=0.71073 \AA, \mu=1.838 \mathrm{~mm}^{-1}$. A black crystal was obtained from $\mathrm{CH}_{2} \mathrm{Cl}_{2}$ /hexane solution at $5{ }^{\circ} \mathrm{C}$. Numbers of measured and unique reflections were 25665 and 5754 , respectively $\left(R_{\text {sigma }}=0.0218\right)$. Final $R(F)=0.0203$ for 302 parameters and 5754 reflections with $I>2 \sigma(I)$ (for all data, $R(F)$ and $w R\left(F^{2}\right)$ values are 0.0243 and 0.0520 , respectively).

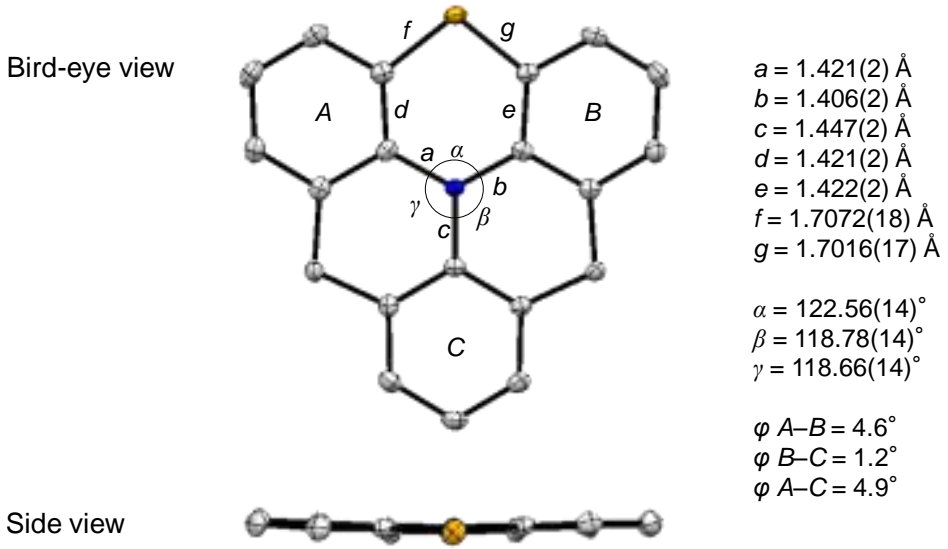

Figure S5. X-ray crystal structure of $\mathbf{4}^{\cdot+} \cdot \mathbf{S b C l}_{\mathbf{6}}{ }^{-}$from bird-eye view (top) and side view (bottom). Displacement ellipsoids are shown at the $50 \%$ probability level. Hydrogen atoms, Me groups, and $\mathrm{SbCl}_{6}{ }^{-}$are omitted for clarity.

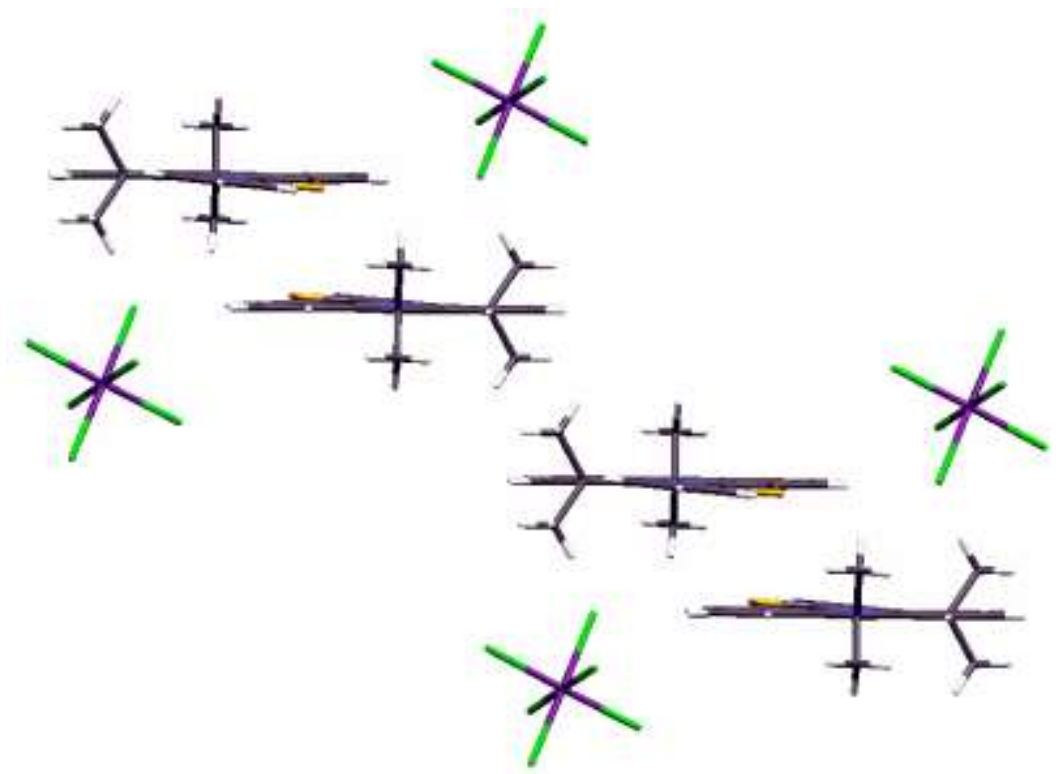

Figure S6. Arrangement of neighboring $\mathbf{4}^{++} \cdot \mathbf{S b C l}_{\mathbf{6}}{ }^{-}$in the crystal packing. 


\section{Photophysical data}

(a)

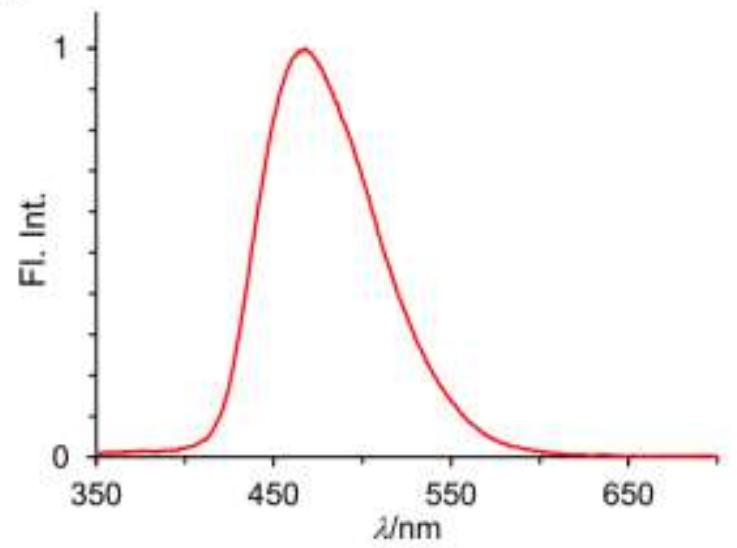

(b)

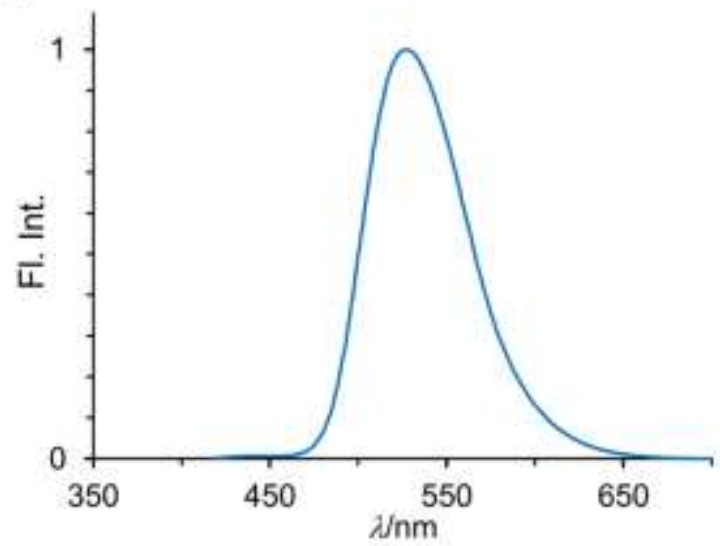

Figure S7. Fluorescence spectra of (a) 4 and (b) 5 in $\mathrm{CH}_{2} \mathrm{Cl}_{2}$. The excitation wavelengths $\lambda_{\mathrm{ex}}=320 \mathrm{~nm}$ and $400 \mathrm{~nm}$ for $\mathbf{4}$ and $\mathbf{5}$, respectively.

Table S1. Photophysical Parameters of 4, 5, and NPPTZ in $\mathrm{CH}_{2} \mathrm{Cl}_{2}$

\begin{tabular}{llccc}
\hline Compd. & \multicolumn{1}{c}{$\lambda_{\max }{ }^{\mathrm{abs}}[\mathrm{nm}]\left(\varepsilon\left[\mathrm{L} \mathrm{mol}^{-1} \mathrm{~cm}^{-1}\right)\right.$} & $\lambda_{\max }{ }^{\mathrm{fl}}[\mathrm{nm}]$ & Stokes shift $\left[\mathrm{cm}^{-1}\right]$ & $\Phi_{\mathrm{f}}{ }^{a}$ \\
\hline $\mathbf{4}$ & $280(42600), 303(15900), 334(4900)$ & 468 & 8570 & 0.02 \\
$\mathbf{5}$ & $261(42600), 327(4600), 447(8400)$ & 527 & 3500 & 0.45 \\
NPPTZ & $258(47400), 320(4500)$ & N/A & N/A & N/A \\
\hline
\end{tabular}

${ }^{a}$ Absolute fluorescence quantum yields determined by an integrating-sphere system.

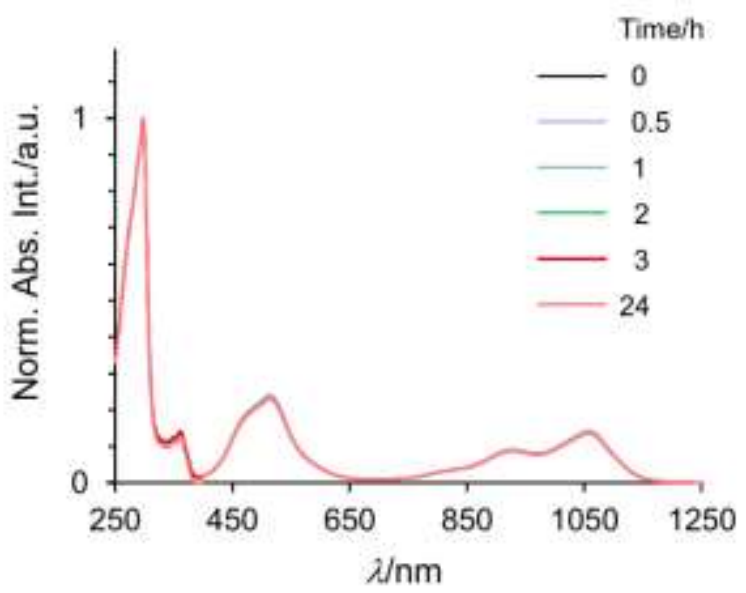

Figure S8. Time profiles of UV-vis-NIR spectra of $4^{\cdot+}$ in $\mathrm{CH}_{2} \mathrm{Cl}_{2}$. 


\section{Electrochemical data}

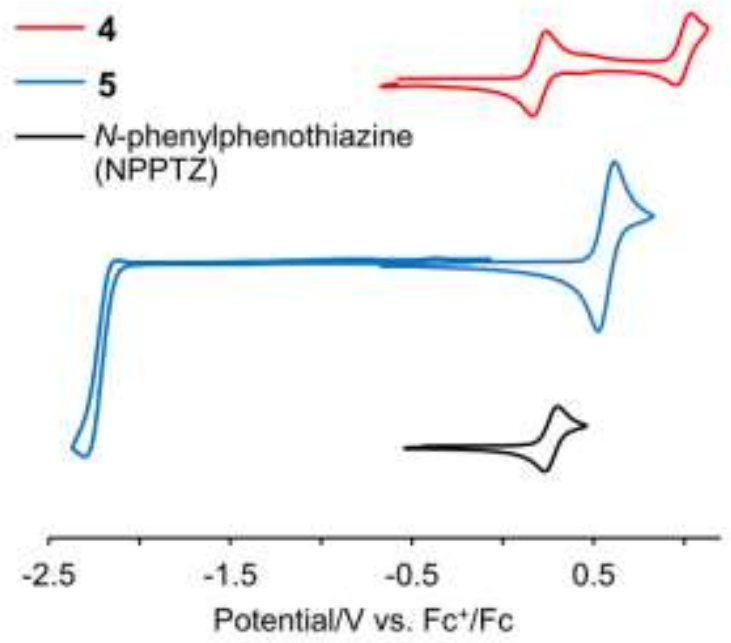

Figure S9. Cyclic voltammograms of $\mathbf{4 , 5}$, and $N$-phenylphenothiazine at scan rate $100 \mathrm{mV} \mathrm{s}^{-1}$ in $\mathrm{CH}_{2} \mathrm{Cl}_{2}\left(0.1 \mathrm{~mol} \mathrm{~L}^{-1}\left[(n-\mathrm{Bu})_{4} \mathrm{~N}\right]\left[\mathrm{PF}_{6}\right]\right)$.

Table S2. Oxidation and Reduction Potentials by CV or DPV in $\mathrm{CH}_{2} \mathrm{Cl}_{2}$ (Supporting Electrolyte: $0.1 \mathrm{~mol} \mathrm{~L}^{-1}\left[(n-\mathrm{Bu})_{4} \mathrm{~N}\right]\left[\mathrm{PF}_{6}\right]$ )

\begin{tabular}{lccc}
\hline Compd. & $E_{\text {ox }}{ }^{1}[\mathrm{~V}]$ & $E_{\text {ox }}{ }^{2}[\mathrm{~V}]$ & $E_{\text {red }}[\mathrm{V}]$ \\
\hline $\mathbf{4}$ & $0.21^{a}$ & $1.00^{a}$ & N/A \\
$\mathbf{5}$ & $0.57^{a}$ & N/A & $-2.20^{b}$ \\
NPPTZ & $0.26^{a}$ & N/A & N/A
\end{tabular}

${ }^{a}$ Reversible wave; the potentials were determined by CV.

${ }^{b}$ Irreversible wave; the potential was determined by DPV. 


\section{Theoretical data}

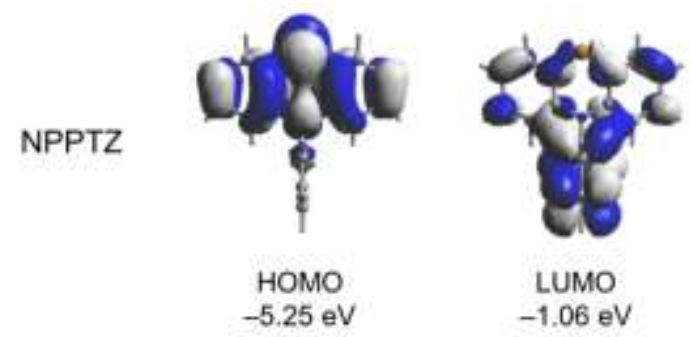

Figure S10. HOMO and LUMO of NPPTZ calculated at the B3LYP/6-311+G(d,p).
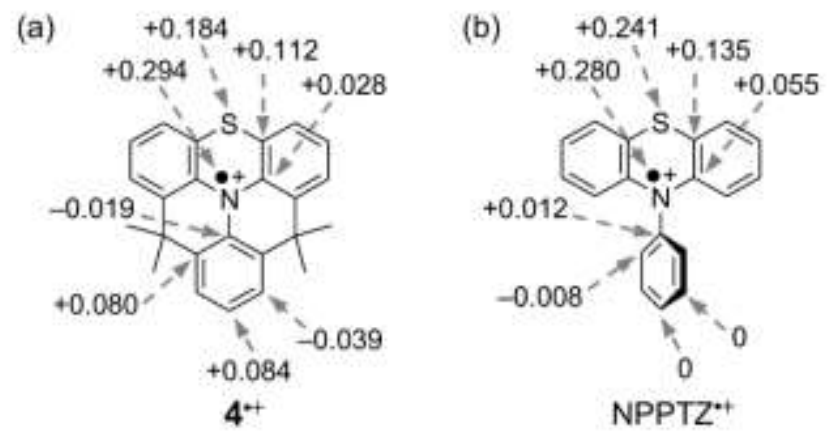

Figure S11. Spin density maps of (a) $4^{\cdot+}$ and (b) NPPTZ ${ }^{+}$calculated at the UB3LYP/6-311+G(d,p).
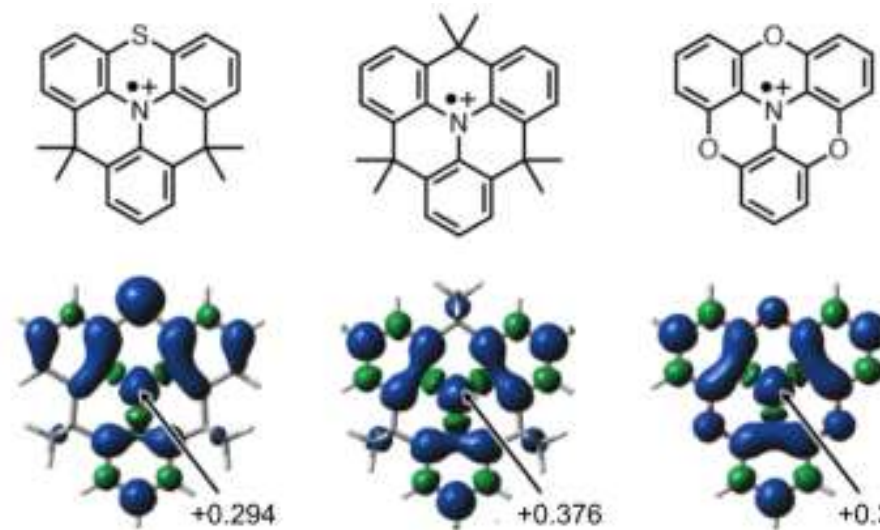

$4^{++}$
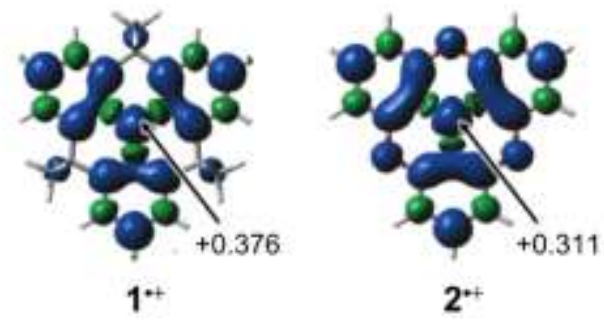

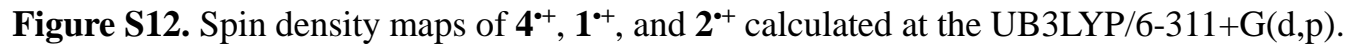


(a)

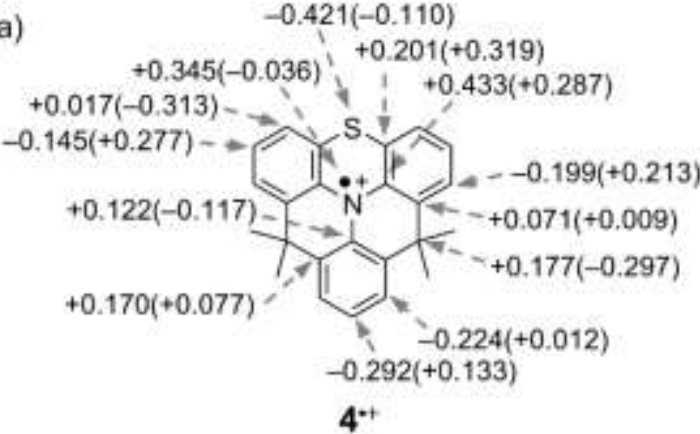

(c)

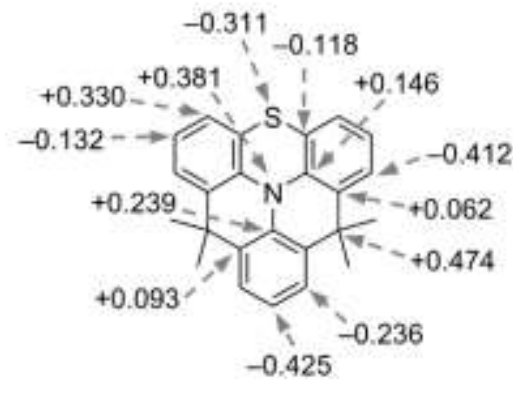

4

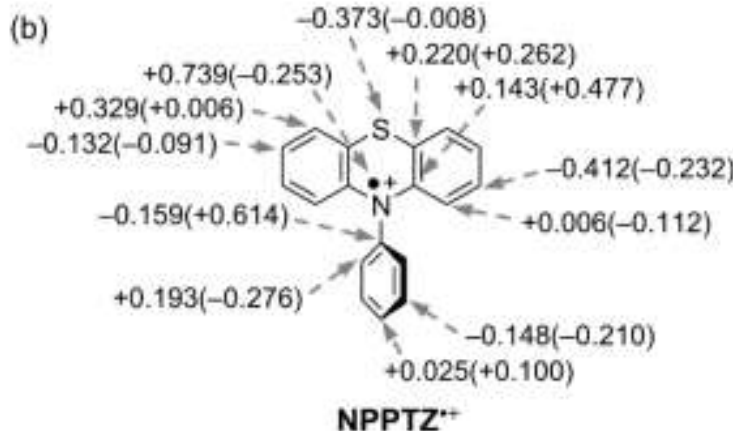

(d)

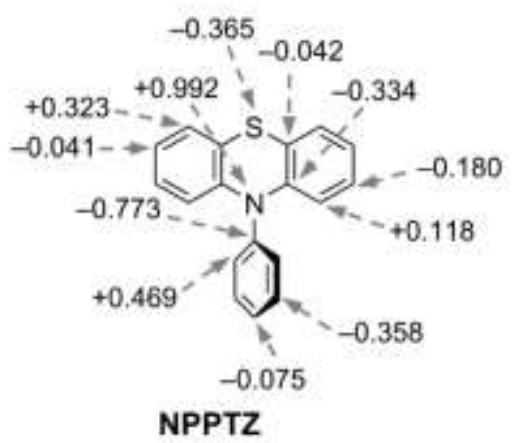

Figure S13. Mulliken charges of (a) $\mathbf{4}^{\cdot+}$ and (b) NPPTZ ${ }^{\circ+}$, (c) 4, and (d) NPPTZ calculated at the (U)B3LYP/6$311+\mathrm{G}(\mathrm{d}, \mathrm{p})$. The values in the parentheses are the differences of Mulliken charges upon oxidation.

Table S3. Selected Data of Electronic Transitions in 4 by the TD-B3LYP/6-311G(d,p)//B3LYP/6-311G(d,p) Level ${ }^{a}$

\begin{tabular}{lccl}
\hline State & $\lambda[\mathrm{nm}(\mathrm{eV})]$ & $f$ & \multicolumn{1}{c}{ Composition of band and CI coefficiencies } \\
\hline 1 & $380(3.27)$ & 0.0954 & $\mathrm{H} \rightarrow \mathrm{L}, 0.69$ \\
2 & $363(3.41)$ & 0.0022 & $\mathrm{H} \rightarrow \mathrm{L}+1,0.69$ \\
3 & $327(3.79)$ & 0.0231 & $\mathrm{H}-1 \rightarrow \mathrm{L}, 0.20 ; \mathrm{H} \rightarrow \mathrm{L}+2,0.66$ \\
4 & $314(3.94)$ & 0.0425 & $\mathrm{H}-1 \rightarrow \mathrm{L}, 0.66 ; \mathrm{H} \rightarrow \mathrm{L}+1,-0.19$ \\
\hline
\end{tabular}

${ }^{a}$ Calculations were carried out for the lowest 20 excited states, and only energies above $280 \mathrm{~nm}$ with $f>$ 0.001 are shown. $f=$ oscillator strength; $\mathrm{CI}=$ configuration interaction; $\mathrm{H}=\mathrm{HOMO} ; \mathrm{L}=\mathrm{LUMO}$.

Table S4. Selected Data of Electronic Transitions in $\mathbf{5}$ by the TD-B3LYP/6-311G(d,p)//B3LYP/6-311G(d,p) Level ${ }^{a}$

\begin{tabular}{lccl}
\hline State & $\lambda[\mathrm{nm}(\mathrm{eV})]$ & $f$ & \multicolumn{1}{c}{ Composition of band and CI coefficiencies } \\
\hline 1 & $409(3.03)$ & 0.0954 & $\mathrm{H} \rightarrow \mathrm{L}, 0.70$ \\
2 & $340(3.64)$ & 0.0345 & $\mathrm{H} \rightarrow \mathrm{L}+1,0.69$ \\
3 & $317(3.91)$ & 0.0423 & $\mathrm{H} \rightarrow \mathrm{L}+2,-0.29 ; \mathrm{H}-1 \rightarrow \mathrm{L}, 0.62$ \\
4 & $301(3.66)$ & 0.1622 & $\mathrm{H} \rightarrow \mathrm{L}+1,0.61 ; \mathrm{H}-2 \rightarrow \mathrm{L},-0.32 ; \mathrm{H} \rightarrow \mathrm{L}, 0.10$ \\
5 & $283(4.38)$ & 0.2296 & $\mathrm{H}-1 \rightarrow \mathrm{L}, 0.62 ; \mathrm{H} \rightarrow \mathrm{L}+2,0.27 ; \mathrm{H} \rightarrow \mathrm{L}+4,0.15$ \\
\hline
\end{tabular}

${ }^{a}$ Calculations were carried out for the lowest 20 excited states, and only energies above $300 \mathrm{~nm}$ with $f>$ 0.001 are shown. $f=$ oscillator strength; $\mathrm{CI}=$ configuration interaction; $\mathrm{H}=\mathrm{HOMO} ; \mathrm{L}=\mathrm{LUMO}$. 
Table S5. Selected Data of Electronic Transitions in NPPTZ by the TD-B3LYP/6-311G(d,p)//B3LYP/6-311G(d,p) Level ${ }^{a}$

\begin{tabular}{lccl}
\hline State & $\lambda[\mathrm{nm}(\mathrm{eV})]$ & $f$ & \multicolumn{1}{c}{ Composition of band and CI coefficiencies } \\
\hline 1 & $322(3.85)$ & 0.0118 & $\mathrm{H} \rightarrow \mathrm{L},-0.15 ; \mathrm{H} \rightarrow \mathrm{L}+1,0.22 ; \mathrm{H} \rightarrow \mathrm{L}+2,0.64$ \\
2 & $306(4.05)$ & 0.0173 & $\mathrm{H} \rightarrow \mathrm{L}+3,0.69$ \\
3 & $293(4.23)$ & 0.0556 & $\mathrm{H}-1 \rightarrow \mathrm{L}, 0.16 ; \mathrm{H}-1 \rightarrow \mathrm{L}+1,0.18 ; \mathrm{H} \rightarrow \mathrm{L}+4,0.66$ \\
\hline
\end{tabular}

${ }^{a}$ Calculations were carried out for the lowest 20 excited states, and only energies above $280 \mathrm{~nm}$ with $f>$ 0.001 are shown. $f=$ oscillator strength; $\mathrm{CI}=$ configuration interaction; $\mathrm{H}=\mathrm{HOMO} ; \mathrm{L}=\mathrm{LUMO}$.

Table S6. Selected Data of Electronic Transitions in $\mathbf{4}^{\cdot+}$ by the TD-UB3LYP/6-311G(d,p)//UB3LYP/6-311G(d,p) Level ${ }^{a}$

\begin{tabular}{lccc}
\hline State & $\lambda[\mathrm{nm}(\mathrm{eV})]$ & $f$ & \multicolumn{1}{c}{ Composition of band and CI coefficiencies } \\
\hline 1 & $980(1.27)$ & 0.0386 & $\mathrm{H}-2 \beta \rightarrow \mathrm{S} \beta,-0.10 ; \mathrm{H} \beta \rightarrow \mathrm{S} \beta, 0.99$ \\
2 & $705(1.76)$ & 0.0022 & $\mathrm{H}-1 \beta \rightarrow \mathrm{S} \beta, 0.99$ \\
3 & $513(2.41)$ & 0.0663 & $\mathrm{H}-2 \beta \rightarrow \mathrm{S} \beta, 0.98$ \\
4 & $464(2.67)$ & 0.1010 & $\mathrm{~S} \alpha \rightarrow \mathrm{L} \alpha,-0.10 ; \mathrm{H}-4 \beta \rightarrow \mathrm{S} \beta, 0.93 ; \mathrm{H}-3 \beta \rightarrow \mathrm{S} \beta, 0.30$ \\
& & $\mathrm{H} \beta \rightarrow \mathrm{L} \beta, 0.13$ \\
\hline
\end{tabular}
0.002 are shown. $f=$ oscillator strength; $\mathrm{CI}=$ configuration interaction; $\mathrm{H}=\mathrm{HOMO} ; \mathrm{L}=\mathrm{LUMO} ; \mathrm{S}=\mathrm{SOMO}$.

Table S7. Selected Data of Electronic Transitions in NPPTZ ${ }^{+}$ by the TD-UB3LYP/6-311G(d,p)//UB3LYP/6-311G(d,p) Level ${ }^{a}$

\begin{tabular}{lccc}
\hline State & $\lambda[\mathrm{nm}(\mathrm{eV})]$ & $f$ & \multicolumn{1}{c}{ Composition of band and CI coefficiencies } \\
\hline 1 & $782(1.59)$ & 0.0162 & $\mathrm{H} \beta \rightarrow \mathrm{S} \beta, 0.99$ \\
2 & $569(2.18)$ & 0.0022 & $\mathrm{H}-4 \beta \rightarrow \mathrm{S} \beta, 0.18 ; \mathrm{H}-1 \beta \rightarrow \mathrm{S} \beta, 0.98$ \\
3 & $478(2.60)$ & 0.0691 & $\mathrm{~S} \alpha \rightarrow \mathrm{L} \alpha, 0.14 ; \mathrm{H}-4 \beta \rightarrow \mathrm{S} \beta,-0.64 ; \mathrm{H}-3 \beta \rightarrow \mathrm{S} \beta, 0.72$ \\
& & & $\mathrm{H} \beta \rightarrow \mathrm{L} \beta, 0.12$ \\
4 & $469(2.64)$ & 0.0687 & $\mathrm{H}-4 \beta \rightarrow \mathrm{S} \beta, 0.72 ; \mathrm{H}-3 \beta \rightarrow \mathrm{S} \beta, 0.65 ; \mathrm{H} \beta \rightarrow \mathrm{L} \beta,-0.13$ \\
\hline
\end{tabular}

${ }^{a}$ Calculations were carried out for the lowest 20 excited states, and only energies above $450 \mathrm{~nm}$ with $f>$ 0.002 are shown. $f=$ oscillator strength; $\mathrm{CI}=$ configuration interaction; $\mathrm{H}=\mathrm{HOMO} ; \mathrm{L}=\mathrm{LUMO} ; \mathrm{S}=\mathrm{SOMO}$. 
Table S8. Cartesian Coordinates of 4 at the B3LYP/6-311+G(d,p) level

\begin{tabular}{|c|c|c|c|c|c|}
\hline \multirow{2}{*}{$\begin{array}{l}\text { Center } \\
\text { Number }\end{array}$} & \multirow{2}{*}{$\begin{array}{l}\text { Atomic } \\
\text { Number }\end{array}$} & \multirow{2}{*}{$\begin{array}{l}\text { Atomic } \\
\text { Type }\end{array}$} & \multicolumn{3}{|c|}{ Coordinates (Angstroms) } \\
\hline & & & $X$ & Y & $\mathrm{Z}$ \\
\hline 1 & 6 & 0 & -2.322564 & -0.149492 & 1.329021 \\
\hline 2 & 6 & 0 & -0.917170 & -0.025123 & 1.245027 \\
\hline 3 & 7 & 0 & -0.229748 & -0.172968 & -0.000000 \\
\hline 4 & 6 & 0 & -0.917170 & -0.025123 & -1.245027 \\
\hline 5 & 6 & 0 & -2.322564 & -0.149492 & -1.329021 \\
\hline 6 & 16 & 0 & -3.253681 & -0.847523 & -0.000000 \\
\hline 7 & 6 & 0 & -3.023159 & 0.151465 & 2.491296 \\
\hline 8 & 6 & 0 & -2.338720 & 0.509163 & 3.644967 \\
\hline 9 & 6 & 0 & -0.955611 & 0.512879 & 3.610613 \\
\hline 10 & 6 & 0 & -0.228968 & 0.233865 & 2.444565 \\
\hline 11 & 6 & 0 & -0.228968 & 0.233865 & -2.444565 \\
\hline 12 & 6 & 0 & -0.955611 & 0.512879 & -3.610613 \\
\hline 13 & 6 & 0 & -2.338720 & 0.509163 & -3.644967 \\
\hline 14 & 6 & 0 & -3.023159 & 0.151465 & -2.491296 \\
\hline 15 & 6 & 0 & 1.196123 & -0.177661 & 0.000000 \\
\hline 16 & 6 & 0 & 1.918260 & -0.184327 & 1.214573 \\
\hline 17 & 6 & 0 & 3.300140 & -0.380331 & 1.187056 \\
\hline 18 & 6 & 0 & 3.999071 & -0.518185 & 0.000000 \\
\hline 19 & 6 & 0 & 3.300140 & -0.380331 & -1.187056 \\
\hline 20 & 6 & 0 & 1.918260 & -0.184327 & -1.214573 \\
\hline 21 & 6 & 0 & 1.283233 & 0.116859 & -2.567025 \\
\hline 22 & 6 & 0 & 1.283233 & 0.116859 & 2.567025 \\
\hline 23 & 6 & 0 & 1.875446 & 1.451561 & -3.098409 \\
\hline 24 & 6 & 0 & 1.600479 & -1.027951 & -3.569142 \\
\hline 25 & 6 & 0 & 1.600479 & -1.027951 & 3.569142 \\
\hline 26 & 6 & 0 & 1.875446 & 1.451561 & 3.098409 \\
\hline 27 & 1 & 0 & -4.104353 & 0.079739 & 2.483242 \\
\hline 28 & 1 & 0 & -2.873679 & 0.747628 & 4.556303 \\
\hline 29 & 1 & 0 & -0.412079 & 0.729509 & 4.521237 \\
\hline 30 & 1 & 0 & -0.412079 & 0.729509 & -4.521237 \\
\hline 31 & 1 & 0 & -2.873679 & 0.747628 & -4.556303 \\
\hline 32 & 1 & 0 & -4.104353 & 0.079739 & -2.483242 \\
\hline 33 & 1 & 0 & 3.845156 & -0.404704 & 2.122086 \\
\hline 34 & 1 & 0 & 5.069634 & -0.685129 & 0.000000 \\
\hline 35 & 1 & 0 & 3.845156 & -0.404704 & -2.122086 \\
\hline 36 & 1 & 0 & 2.959681 & 1.381294 & -3.206549 \\
\hline 37 & 1 & 0 & 1.656133 & 2.270317 & -2.409239 \\
\hline 38 & 1 & 0 & 1.458624 & 1.708022 & -4.074611 \\
\hline 39 & 1 & 0 & 2.676693 & -1.148343 & -3.706402 \\
\hline 40 & 1 & 0 & 1.163862 & -0.823125 & -4.548465 \\
\hline 41 & 1 & 0 & 1.194946 & -1.976074 & -3.208747 \\
\hline 42 & 1 & 0 & 2.676693 & -1.148343 & 3.706402 \\
\hline 43 & 1 & 0 & 1.194946 & -1.976074 & 3.208747 \\
\hline 44 & 1 & 0 & 1.163862 & -0.823125 & 4.548465 \\
\hline 45 & 1 & 0 & 2.959681 & 1.381294 & 3.206549 \\
\hline 46 & 1 & 0 & 1.458624 & 1.708022 & 4.074611 \\
\hline 47 & 1 & 0 & 1.656133 & 2.270317 & 2.409239 \\
\hline
\end{tabular}

No imaginary frequency.

Total energy $=-1380.41980117$ hartree.

$\mathrm{HOMO}=-0.18784$ hartree; LUMO $=-0.03946$ hartree

Table S9. Cartesian Coordinates of $\mathbf{4}^{\cdot+}$ at the UB3LYP/6-311+G(d,p) level

\begin{tabular}{|c|c|c|c|c|c|}
\hline \multirow{2}{*}{$\begin{array}{l}\text { Center } \\
\text { Number }\end{array}$} & \multirow{2}{*}{$\begin{array}{l}\text { Atomic } \\
\text { Number }\end{array}$} & \multirow{2}{*}{$\begin{array}{l}\text { Atomic } \\
\text { Type }\end{array}$} & \multicolumn{3}{|c|}{ Coordinates (Angstroms) } \\
\hline & & & $X$ & $Y$ & $Z$ \\
\hline 1 & 6 & 0 & -2.338960 & -0.011558 & 1.343422 \\
\hline 2 & 6 & 0 & -0.916293 & -0.000262 & 1.250090 \\
\hline 3 & 7 & 0 & -0.230669 & -0.007094 & 0.000000 \\
\hline 4 & 6 & 0 & -0.916293 & -0.000262 & -1.250090 \\
\hline 5 & 6 & 0 & -2.338960 & -0.011558 & -1.343422 \\
\hline 6 & 16 & 0 & -3.422463 & -0.063366 & 0.000000 \\
\hline
\end{tabular}




$\begin{array}{rrrrrr}7 & 6 & 0 & -3.014525 & 0.008610 & 2.569907 \\ 8 & 6 & 0 & -2.307919 & 0.038289 & 3.747993 \\ 9 & 6 & 0 & -0.918910 & 0.040810 & 3.681886 \\ 10 & 6 & 0 & -0.212786 & 0.018870 & 2.483269 \\ 11 & 6 & 0 & -0.212786 & 0.018870 & -2.483269 \\ 12 & 6 & 0 & -0.918910 & 0.040810 & -3.681886 \\ 13 & 6 & 0 & -2.307919 & 0.038289 & -3.747993 \\ 14 & 6 & 0 & -3.014525 & 0.008610 & -2.569907 \\ 15 & 6 & 0 & 1.214689 & -0.011306 & -0.000000 \\ 16 & 6 & 0 & 1.936862 & -0.014222 & 1.218077 \\ 17 & 6 & 0 & 3.329917 & -0.032642 & 1.187301 \\ 18 & 6 & 0 & 4.038129 & -0.044740 & -0.000000 \\ 19 & 6 & 0 & 3.329917 & -0.032642 & -1.187301 \\ 20 & 6 & 0 & 1.936862 & -0.014222 & -1.218077 \\ 21 & 6 & 0 & 1.297327 & 0.009454 & -2.589444 \\ 22 & 6 & 0 & 1.297327 & 0.009454 & 2.589444 \\ 23 & 6 & 0 & 1.759518 & 1.291776 & -3.341034 \\ 24 & 6 & 0 & 1.735949 & -1.259272 & -3.377735 \\ 25 & 6 & 0 & 1.735949 & -1.259272 & 3.377735 \\ 26 & 6 & 0 & 1.759518 & 1.291776 & 3.341034 \\ 27 & 1 & 0 & -4.098477 & 0.002013 & 2.570824 \\ 28 & 1 & 0 & -2.818138 & 0.057424 & 4.702641 \\ 29 & 1 & 0 & -0.362171 & 0.059141 & 4.608002 \\ 30 & 1 & 0 & -0.362171 & 0.059141 & -4.608002 \\ 31 & 1 & 0 & -2.818138 & 0.057424 & -4.702641 \\ 32 & 1 & 0 & -4.098477 & 0.002013 & -2.570824 \\ 33 & 1 & 0 & 3.874842 & -0.035490 & 2.120949 \\ 34 & 1 & 0 & 5.121049 & -0.059775 & -0.000000 \\ 35 & 1 & 0 & 3.874842 & -0.035490 & -2.120949 \\ 36 & 1 & 0 & 2.843897 & 1.309538 & -3.451910 \\ 37 & 1 & 0 & 1.458862 & 2.190543 & -2.798058 \\ 38 & 1 & 0 & 1.327224 & 1.337935 & -4.341458 \\ 39 & 1 & 0 & 2.819554 & -1.293402 & -3.491827 \\ 40 & 1 & 0 & 1.301231 & -1.269524 & -4.378022 \\ 41 & 1 & 0 & 1.420171 & -2.167517 & -2.859707 \\ 42 & 1 & 0 & 2.819554 & -1.293402 & 3.491827 \\ 43 & 1 & 0 & 1.420171 & -2.167517 & 2.859707 \\ 44 & 1 & 0 & 1.301231 & -1.269524 & 4.378022 \\ 46 & 1 & 0 & 2.843897 & 1.309538 & 3.451910 \\ 47 & 1 & 0 & 1.327224 & 1.337935 & 4.341458 \\ & 1 & 0 & 1.458862 & 2.190543 & 2.798058\end{array}$

No imaginary frequency.

Total energy $=-1380.18855081$ hartree.

Table S10. Cartesian Coordinates of NPPTZ at the B3LYP/6-311+G(d,p) level

\begin{tabular}{|c|c|c|c|c|c|}
\hline \multirow{2}{*}{$\begin{array}{l}\text { Center } \\
\text { Number }\end{array}$} & \multirow{2}{*}{$\begin{array}{l}\text { Atomic } \\
\text { Number }\end{array}$} & \multirow{2}{*}{$\begin{array}{l}\text { Atomic } \\
\text { Type }\end{array}$} & \multicolumn{3}{|c|}{ Coordinates (Angstroms) } \\
\hline & & & $X$ & $\mathrm{Y}$ & \\
\hline 1 & 6 & 0 & 1.795566 & -1.353377 & 0.173761 \\
\hline 2 & 6 & 0 & 0.417415 & -1.236581 & -0.083922 \\
\hline 3 & 7 & 0 & -0.248972 & 0.000127 & 0.119916 \\
\hline 4 & 6 & 0 & 0.418047 & 1.236507 & -0.083960 \\
\hline 5 & 6 & 0 & 1.796266 & 1.352577 & 0.173722 \\
\hline 6 & 16 & 0 & 2.678422 & -0.000620 & 0.920780 \\
\hline 7 & 6 & 0 & 2.464716 & -2.553580 & -0.055717 \\
\hline 8 & 6 & 0 & 1.771489 & -3.681168 & -0.489855 \\
\hline 9 & 6 & 0 & 0.401922 & -3.587218 & -0.712192 \\
\hline 10 & 6 & 0 & -0.265859 & -2.378455 & -0.525919 \\
\hline 11 & 6 & 0 & -0.264606 & 2.378763 & -0.525950 \\
\hline 12 & 6 & 0 & 0.403828 & 3.587163 & -0.712242 \\
\hline 13 & 6 & 0 & 1.773446 & 3.680373 & -0.489929 \\
\hline 14 & 6 & 0 & 2.466061 & 2.552417 & -0.055781 \\
\hline 15 & 6 & 0 & -1.688476 & 0.000419 & 0.156273 \\
\hline 16 & 6 & 0 & -2.447438 & 0.000336 & -1.016969 \\
\hline 17 & 6 & 0 & -3.839118 & 0.000573 & -0.946942 \\
\hline 18 & 6 & 0 & -4.477402 & 0.000873 & 0.2931 \\
\hline
\end{tabular}




$\begin{array}{rrrrrr}19 & 6 & 0 & -3.720214 & 0.000909 & 1.463148 \\ 20 & 6 & 0 & -2.327669 & 0.000672 & 1.395615 \\ 21 & 1 & 0 & 3.531647 & -2.603500 & 0.130341 \\ 22 & 1 & 0 & 2.296718 & -4.615661 & -0.646615 \\ 23 & 1 & 0 & -0.159380 & -4.453504 & -1.043204 \\ 24 & 1 & 0 & -1.329232 & -2.328972 & -0.712706 \\ 25 & 1 & 0 & -1.328005 & 2.329879 & -0.712711 \\ 26 & 1 & 0 & -0.157009 & 4.453752 & -1.043248 \\ 27 & 1 & 0 & 2.299179 & 4.614582 & -0.646702 \\ 28 & 1 & 0 & 3.533019 & 2.601765 & 0.130274 \\ 29 & 1 & 0 & -1.945218 & 0.000081 & -1.977676 \\ 30 & 1 & 0 & -4.423881 & 0.000518 & -1.859905 \\ 31 & 1 & 0 & -5.560173 & 0.001054 & 0.346220 \\ 32 & 1 & 0 & -4.211519 & 0.001123 & 2.429501 \\ 33 & 1 & 0 & -1.725937 & 0.000693 & 2.296886\end{array}$

No imaginary frequency.

Total energy $=-1146.89235639$ hartree.

$\mathrm{HOMO}=-0.19295$ hartree; LUMO $=-0.03891$ hartree.

Table S11. Cartesian Coordinates of $\mathrm{NPPTZ}^{+}$at the UB3LYP/6-311+G(d,p) level

\begin{tabular}{|c|c|c|c|c|c|}
\hline \multirow{2}{*}{$\begin{array}{l}\text { Center } \\
\text { Number }\end{array}$} & \multirow{2}{*}{$\begin{array}{l}\text { Atomic } \\
\text { Number }\end{array}$} & \multirow{2}{*}{$\begin{array}{l}\text { Atomic } \\
\text { Type }\end{array}$} & \multicolumn{3}{|c|}{ Coordinates (Angstroms) } \\
\hline & & & $\mathrm{X}$ & $\mathrm{Y}$ & Z \\
\hline 1 & 6 & 0 & -1.831135 & 1.361848 & 0.000029 \\
\hline 2 & 6 & 0 & -0.416196 & 1.239161 & -0.000146 \\
\hline 3 & 7 & 0 & 0.227243 & -0.000019 & -0.000118 \\
\hline 4 & 6 & 0 & -0.416306 & -1.239145 & -0.000151 \\
\hline 5 & 6 & 0 & -1.831258 & -1.361703 & 0.000024 \\
\hline 6 & 16 & 0 & -2.913744 & 0.000121 & 0.000063 \\
\hline 7 & 6 & 0 & -2.438333 & 2.628999 & 0.000099 \\
\hline 8 & 6 & 0 & -1.668470 & 3.773215 & -0.000036 \\
\hline 9 & 6 & 0 & -0.267976 & 3.664895 & -0.000256 \\
\hline 10 & 6 & 0 & 0.346175 & 2.430290 & -0.000294 \\
\hline 11 & 6 & 0 & 0.345953 & -2.430350 & -0.000300 \\
\hline 12 & 6 & 0 & -0.268315 & -3.664897 & -0.000264 \\
\hline 13 & 6 & 0 & -1.668818 & -3.773086 & -0.000045 \\
\hline 14 & 6 & 0 & -2.438572 & -2.628799 & 0.000092 \\
\hline 15 & 6 & 0 & 1.686610 & -0.000069 & 0.000020 \\
\hline 16 & 6 & 0 & 2.366624 & -0.000081 & -1.215256 \\
\hline 17 & 6 & 0 & 3.760457 & -0.000129 & -1.208685 \\
\hline 18 & 6 & 0 & 4.455250 & -0.000159 & 0.000371 \\
\hline 19 & 6 & 0 & 3.760146 & -0.000138 & 1.209259 \\
\hline 20 & 6 & 0 & 2.366323 & -0.000090 & 1.215477 \\
\hline 21 & 1 & 0 & -3.520259 & 2.695334 & 0.000263 \\
\hline 22 & 1 & 0 & -2.142939 & 4.746323 & 0.000029 \\
\hline 23 & 1 & 0 & 0.342966 & 4.559076 & -0.000397 \\
\hline 24 & 1 & 0 & 1.423773 & 2.371176 & -0.000431 \\
\hline 25 & 1 & 0 & 1.423555 & -2.371342 & -0.000435 \\
\hline 26 & 1 & 0 & 0.342543 & -4.559135 & -0.000406 \\
\hline 27 & 1 & 0 & -2.143379 & -4.746149 & 0.000018 \\
\hline 28 & 1 & 0 & -3.520504 & -2.695032 & 0.000256 \\
\hline 29 & 1 & 0 & 1.816211 & -0.000059 & -2.148850 \\
\hline 30 & 1 & 0 & 4.300297 & -0.000144 & -2.147795 \\
\hline 31 & 1 & 0 & 5.538558 & -0.000196 & 0.000513 \\
\hline 32 & 1 & 0 & 4.299759 & -0.000159 & 2.148500 \\
\hline 33 & 1 & 0 & 1.815659 & -0.000074 & 2.148924 \\
\hline
\end{tabular}

No imaginary frequency.

Total energy $=-1146.65675117$ hartree .

Table S12. Cartesian Coordinates of 5 at the B3LYP/6-311+G(d,p) level

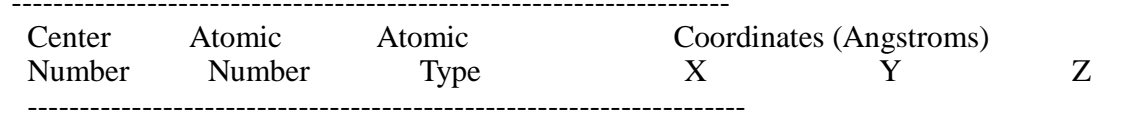




\begin{tabular}{|c|c|c|c|c|c|}
\hline 1 & 6 & 0 & -2.228748 & -1.522172 & -0.100258 \\
\hline 2 & 6 & 0 & -1.617070 & -0.249339 & -0.006375 \\
\hline 3 & 6 & 0 & -2.486609 & 0.843810 & 0.202144 \\
\hline 4 & 6 & 0 & -3.861511 & 0.654663 & 0.424274 \\
\hline 5 & 6 & 0 & -4.411623 & -0.607214 & 0.435443 \\
\hline 6 & 6 & 0 & -3.581976 & -1.695245 & 0.156679 \\
\hline 7 & 16 & 0 & -1.352395 & -2.924359 & -0.714056 \\
\hline 8 & 6 & 0 & 0.260482 & -2.506662 & -0.129240 \\
\hline 9 & 6 & 0 & 0.702648 & -1.169895 & -0.023284 \\
\hline 10 & 7 & 0 & -0.215683 & -0.064914 & -0.130677 \\
\hline 11 & 6 & 0 & 1.111111 & -3.581138 & 0.110007 \\
\hline 12 & 6 & 0 & 2.449840 & -3.361973 & 0.398617 \\
\hline 13 & 6 & 0 & 2.915557 & -2.059826 & 0.410321 \\
\hline 14 & 6 & 0 & 2.076210 & -0.958579 & 0.190251 \\
\hline 15 & 6 & 0 & 0.302118 & 1.251653 & -0.135026 \\
\hline 16 & 6 & 0 & 1.694250 & 1.499694 & -0.132235 \\
\hline 17 & 6 & 0 & 2.141589 & 2.810765 & -0.277999 \\
\hline 18 & 6 & 0 & 1.279404 & 3.900659 & -0.382768 \\
\hline 19 & 6 & 0 & -0.074561 & 3.672630 & -0.276857 \\
\hline 20 & 6 & 0 & -0.568611 & 2.369742 & -0.136204 \\
\hline 21 & 6 & 0 & -2.008164 & 2.225916 & 0.097225 \\
\hline 22 & 6 & 0 & 2.732376 & 0.414000 & 0.112071 \\
\hline 23 & 6 & 0 & 3.760612 & 0.398846 & -1.052574 \\
\hline 24 & 6 & 0 & 3.460839 & 0.723472 & 1.448956 \\
\hline 25 & 8 & 0 & -2.761266 & 3.190619 & 0.194780 \\
\hline 26 & 1 & 0 & -4.457873 & 1.545319 & 0.574400 \\
\hline 27 & 1 & 0 & -5.466928 & -0.758767 & 0.626690 \\
\hline 28 & 1 & 0 & -3.991860 & -2.697880 & 0.109438 \\
\hline 29 & 1 & 0 & 0.711903 & -4.586858 & 0.052027 \\
\hline 30 & 1 & 0 & 3.118166 & -4.192995 & 0.588831 \\
\hline 31 & 1 & 0 & 3.967871 & -1.883419 & 0.589816 \\
\hline 32 & 1 & 0 & 3.208749 & 2.994960 & -0.290843 \\
\hline 33 & 1 & 0 & 1.672708 & 4.902671 & -0.504833 \\
\hline 34 & 1 & 0 & -0.799742 & 4.476378 & -0.278777 \\
\hline 35 & 1 & 0 & 4.265889 & 1.362271 & -1.142955 \\
\hline 36 & 1 & 0 & 3.263924 & 0.185250 & -2.001699 \\
\hline 37 & 1 & 0 & 4.527690 & -0.360912 & -0.892119 \\
\hline 38 & 1 & 0 & 3.951554 & 1.697946 & 1.406480 \\
\hline 39 & 1 & 0 & 4.225343 & -0.025101 & 1.666074 \\
\hline 40 & 1 & 0 & 2.751858 & 0.737490 & 2.279861 \\
\hline
\end{tabular}

No imaginary frequency.

Total energy $=-1375.83385539$ hartree.

$\mathrm{HOMO}=-0.20472$ hartree; $\mathrm{LUMO}=-0.07813$ hartree .

Table S13. Cartesian Coordinates of $\mathbf{1}^{\text {•+ }}$ at the UB3LYP/6-311+G(d,p) level

\begin{tabular}{rrrrrr} 
Center & Atomic & Atomic & \multicolumn{2}{c}{ Coordinates (Angstroms) } \\
Number & Number & Type & X & Y & $Z$ \\
-1 & 7 & 0 & 0.000024 & 0.000030 & -0.000003 \\
2 & 6 & 0 & -1.239588 & -0.704068 & -0.000009 \\
3 & 6 & 0 & -1.262594 & -2.124520 & 0.000270 \\
4 & 6 & 0 & -2.488008 & -2.783794 & 0.000252 \\
5 & 6 & 0 & -3.689710 & -2.096051 & -0.000177 \\
6 & 1 & 0 & -4.631591 & -2.630986 & -0.000258 \\
7 & 6 & 0 & -3.664912 & -0.711710 & -0.000481 \\
8 & 1 & 0 & -4.601887 & -0.172624 & -0.000807 \\
9 & 6 & 0 & -2.471404 & 0.003622 & -0.000314 \\
10 & 6 & 0 & 1.229650 & -0.721381 & -0.000058 \\
11 & 6 & 0 & 1.232748 & -2.141991 & 0.000118 \\
12 & 6 & 0 & 2.448847 & -2.818364 & -0.000070 \\
13 & 1 & 0 & 2.450469 & -3.899428 & 0.000142 \\
14 & 6 & 0 & 3.660051 & -2.147573 & -0.000506 \\
15 & 1 & 0 & 4.594337 & -2.695659 & -0.000708 \\
16 & 6 & 0 & 3.654611 & -0.763015 & -0.000629 \\
17 & 1 & 0 & 4.599054 & -0.237134 & -0.000913 \\
18 & 6 & 0 & 2.471243 & -0.031025 & -0.000332
\end{tabular}




$\begin{array}{rrrrrr}19 & 6 & 0 & 0.010012 & 1.425519 & 0.000014 \\ 20 & 6 & 0 & 1.238690 & 2.138601 & 0.000099 \\ 21 & 6 & 0 & 1.216214 & 3.529894 & 0.000343 \\ 22 & 1 & 0 & 2.151808 & 4.071599 & 0.000427 \\ 23 & 6 & 0 & 0.029731 & 4.243599 & 0.000419 \\ 24 & 1 & 0 & 0.037276 & 5.326749 & 0.000605 \\ 25 & 6 & 0 & -1.166610 & 3.546544 & 0.000187 \\ 26 & 1 & 0 & -2.094468 & 4.101342 & 0.000133 \\ 27 & 6 & 0 & -1.208609 & 2.155727 & -0.000020 \\ 28 & 1 & 0 & -2.504686 & -3.864746 & 0.000563 \\ 29 & 6 & 0 & -2.575958 & 1.511481 & -0.000432 \\ 30 & 6 & 0 & 2.596866 & 1.475299 & -0.000211 \\ 31 & 6 & 0 & -3.349057 & 1.964437 & 1.274530 \\ 32 & 1 & 0 & -3.460241 & 3.048822 & 1.298112 \\ 33 & 1 & 0 & -4.348884 & 1.530092 & 1.298281 \\ 34 & 1 & 0 & -2.821436 & 1.655832 & 2.179599 \\ 35 & 6 & 0 & -3.348151 & 1.964337 & -1.275978 \\ 36 & 1 & 0 & -4.347944 & 1.529942 & -1.300412 \\ 37 & 1 & 0 & -3.459327 & 3.048724 & -1.299680 \\ 38 & 1 & 0 & -2.819872 & 1.655702 & -2.180652 \\ 39 & 6 & 0 & 3.375923 & 1.917265 & 1.274982 \\ 40 & 1 & 0 & 4.369747 & 1.469342 & 1.298754 \\ 41 & 1 & 0 & 3.501861 & 3.000025 & 1.299023 \\ 42 & 1 & 0 & 2.844042 & 1.615545 & 2.179882 \\ 43 & 6 & 0 & 3.375402 & 1.917586 & -1.275607 \\ 44 & 1 & 0 & 3.501304 & 3.000357 & -1.299417 \\ 45 & 1 & 0 & 4.369225 & 1.469684 & -1.299894 \\ 46 & 1 & 0 & 2.843159 & 1.616089 & -2.180368 \\ 47 & 6 & 0 & -0.020885 & -2.986237 & 0.000711 \\ 48 & 6 & 0 & -0.027095 & -3.880966 & 1.276588 \\ 49 & 1 & 0 & -0.911307 & -4.518408 & 1.300757 \\ 50 & 1 & 0 & 0.848137 & -4.530676 & 1.300781 \\ 51 & 1 & 0 & -0.022850 & -3.268826 & 2.181036 \\ 52 & 6 & 0 & -0.027294 & -3.882718 & -1.273906 \\ 53 & 1 & 0.847911 & -4.532475 & -1.297410 \\ 54 & 0 & -0.911448 & -4.520265 & -1.297081 \\ 55 & 1 & 0 & -0.023234 & -3.271813 & -2.179189\end{array}$

No imaginary frequency.

Total energy $=-1099.95338090$ hartree.

Table S14. Cartesian Coordinates of $\mathbf{2}^{\cdot+}$ at the UB3LYP/6-311+G(d,p) level

\begin{tabular}{rrrrrr} 
Center & Atomic & Atomic & \multicolumn{2}{c}{ Coordinates (Angstroms) } \\
Number & Number & Type & X & Y & $Z$ \\
-1 & 7 & 0 & -0.000066 & 0.000076 & -0.000787 \\
2 & 6 & 0 & -1.151749 & -0.763617 & -0.000418 \\
3 & 6 & 0 & -1.053364 & -2.164579 & -0.000139 \\
4 & 6 & 0 & -2.206016 & -2.930983 & 0.000394 \\
5 & 6 & 0 & -3.444424 & -2.284298 & 0.000655 \\
6 & 1 & 0 & -4.346737 & -2.882870 & 0.001089 \\
7 & 6 & 0 & -3.558555 & -0.891873 & 0.000404 \\
8 & 1 & 0 & -4.523195 & -0.401771 & 0.000614 \\
9 & 6 & 0 & -2.404176 & -0.128006 & -0.000125 \\
10 & 8 & 0 & 0.172495 & -2.779343 & -0.000561 \\
11 & 6 & 0 & 1.237271 & -0.615490 & -0.000428 \\
12 & 6 & 0 & 1.312855 & -2.017801 & -0.000159 \\
13 & 6 & 0 & 2.551540 & -2.635703 & 0.000342 \\
14 & 1 & 0 & 2.609315 & -3.716161 & 0.000530 \\
15 & 6 & 0 & 3.700446 & -1.840811 & 0.000598 \\
16 & 1 & 0 & 4.669953 & -2.323031 & 0.001005 \\
17 & 6 & 0 & 3.641536 & -0.445040 & 0.000374 \\
18 & 1 & 0 & 4.538087 & 0.160730 & 0.000590 \\
19 & 6 & 0 & 2.401455 & 0.170088 & -0.000122 \\
20 & 8 & 0 & 2.321016 & 1.538990 & -0.000449 \\
21 & 6 & 0 & -0.085589 & 1.379262 & -0.000426 \\
22 & 6 & 0 & 1.091178 & 2.145845 & -0.000133
\end{tabular}




$\begin{array}{rrrrrr}23 & 6 & 0 & 1.007000 & 3.527447 & 0.000346 \\ 24 & 1 & 0 & 1.913849 & 4.117646 & 0.000552 \\ 25 & 6 & 0 & -0.255948 & 4.124916 & 0.000558 \\ 26 & 1 & 0 & -0.323098 & 5.205639 & 0.000947 \\ 27 & 6 & 0 & -1.435353 & 3.376010 & 0.000321 \\ 28 & 1 & 0 & -2.408268 & 3.849515 & 0.000510 \\ 29 & 6 & 0 & -1.348147 & 1.994590 & -0.000159 \\ 30 & 8 & 0 & -2.493482 & 1.240392 & -0.000517 \\ 31 & 1 & 0 & -2.129430 & -4.010295 & 0.000597\end{array}$

No imaginary frequency.

Total energy $=-971.74973800$ hartree . 


\section{7. ${ }^{1} \mathrm{H}$ and ${ }^{13} \mathrm{C}$ NMR spectral data}

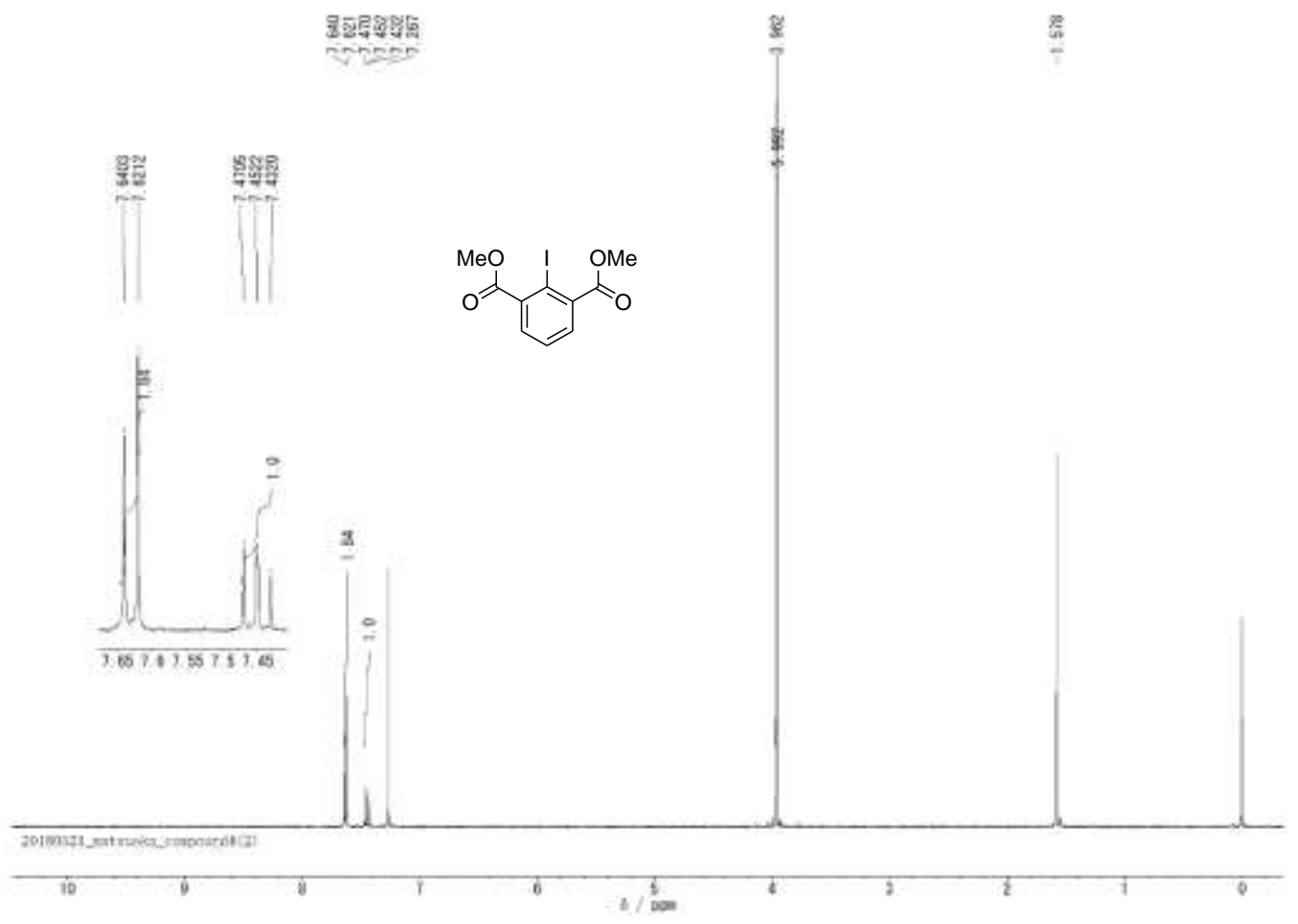

${ }^{1} \mathrm{H}$ NMR spectrum of 6 in $\mathrm{CDCl}_{3}$ solution (400 MHz). 


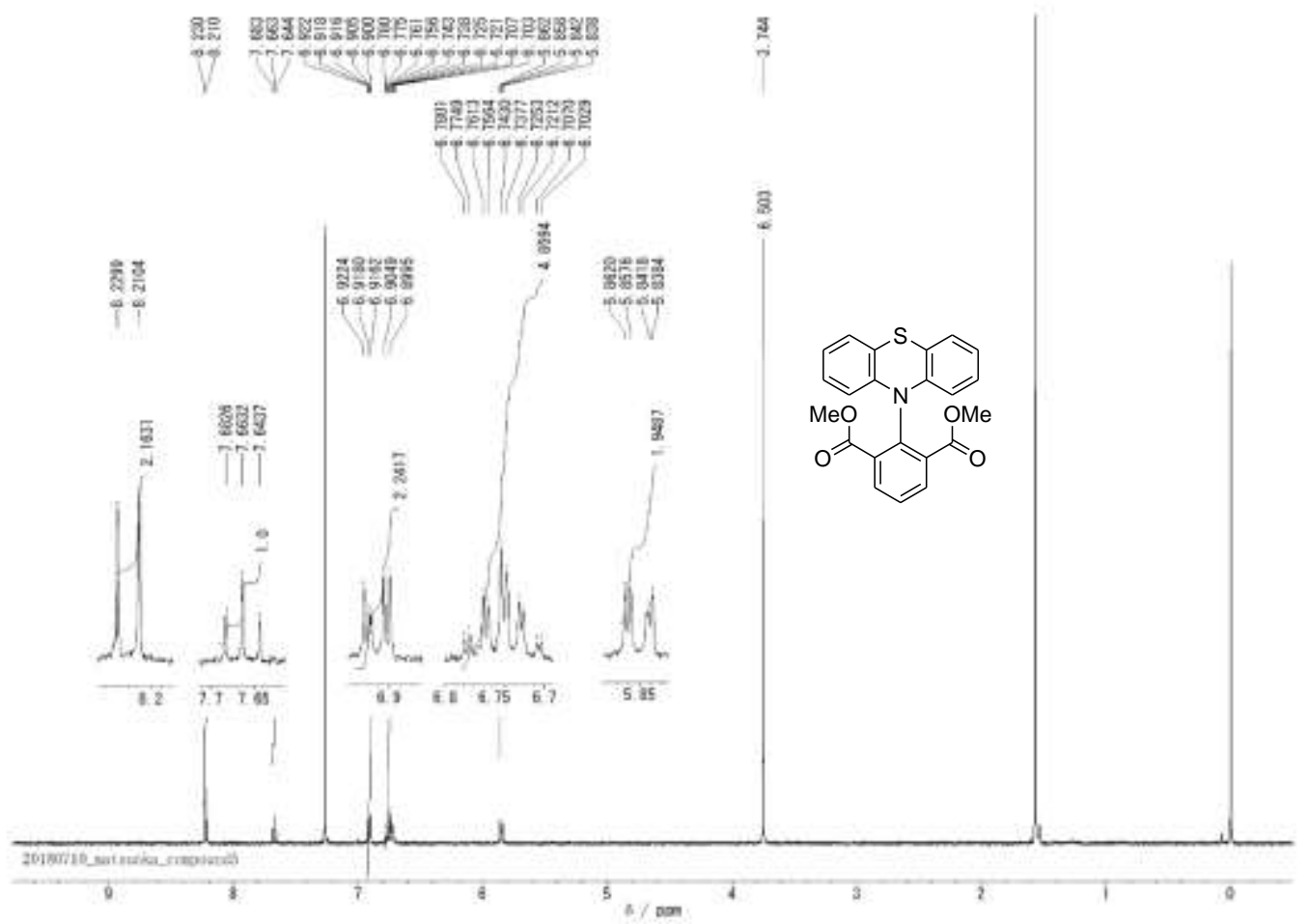

${ }^{1} \mathrm{H}$ NMR spectrum of 7 in $\mathrm{CDCl}_{3}$ solution $(400 \mathrm{MHz})$.

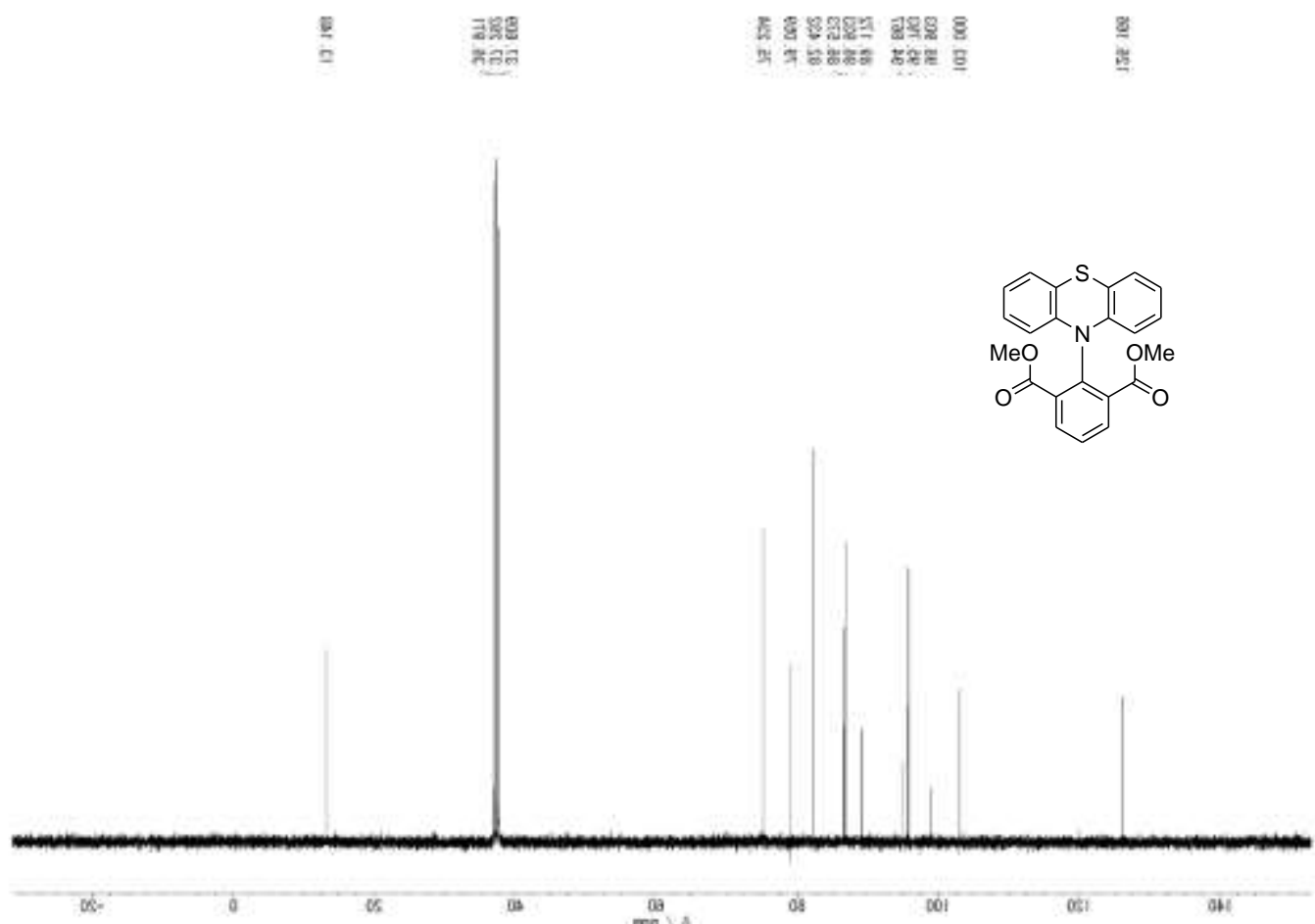

${ }^{13} \mathrm{C}$ NMR spectrum of 7 in $\mathrm{CDCl}_{3}$ solution $(100 \mathrm{MHz})$. 


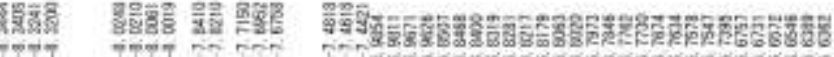

Tiv
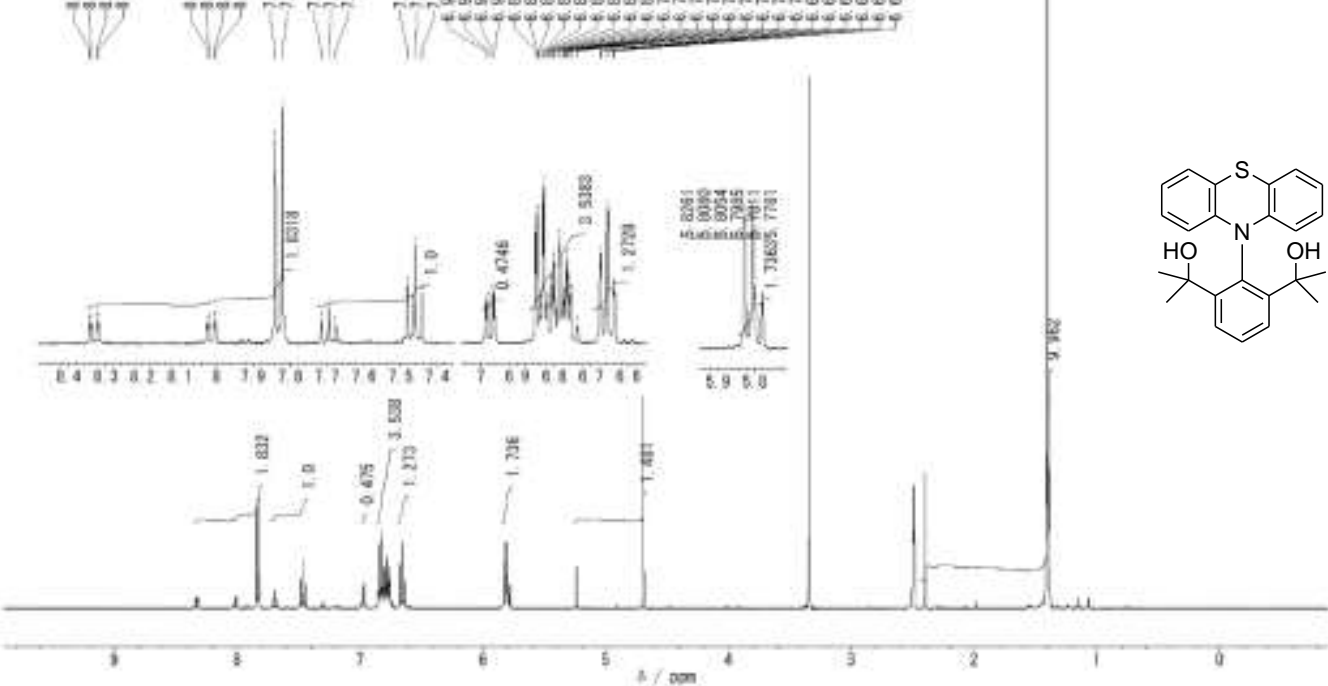

${ }^{1} \mathrm{H}$ NMR spectrum of 8 (DMSO- $\left.d_{6}, 400 \mathrm{MHz}\right)$.

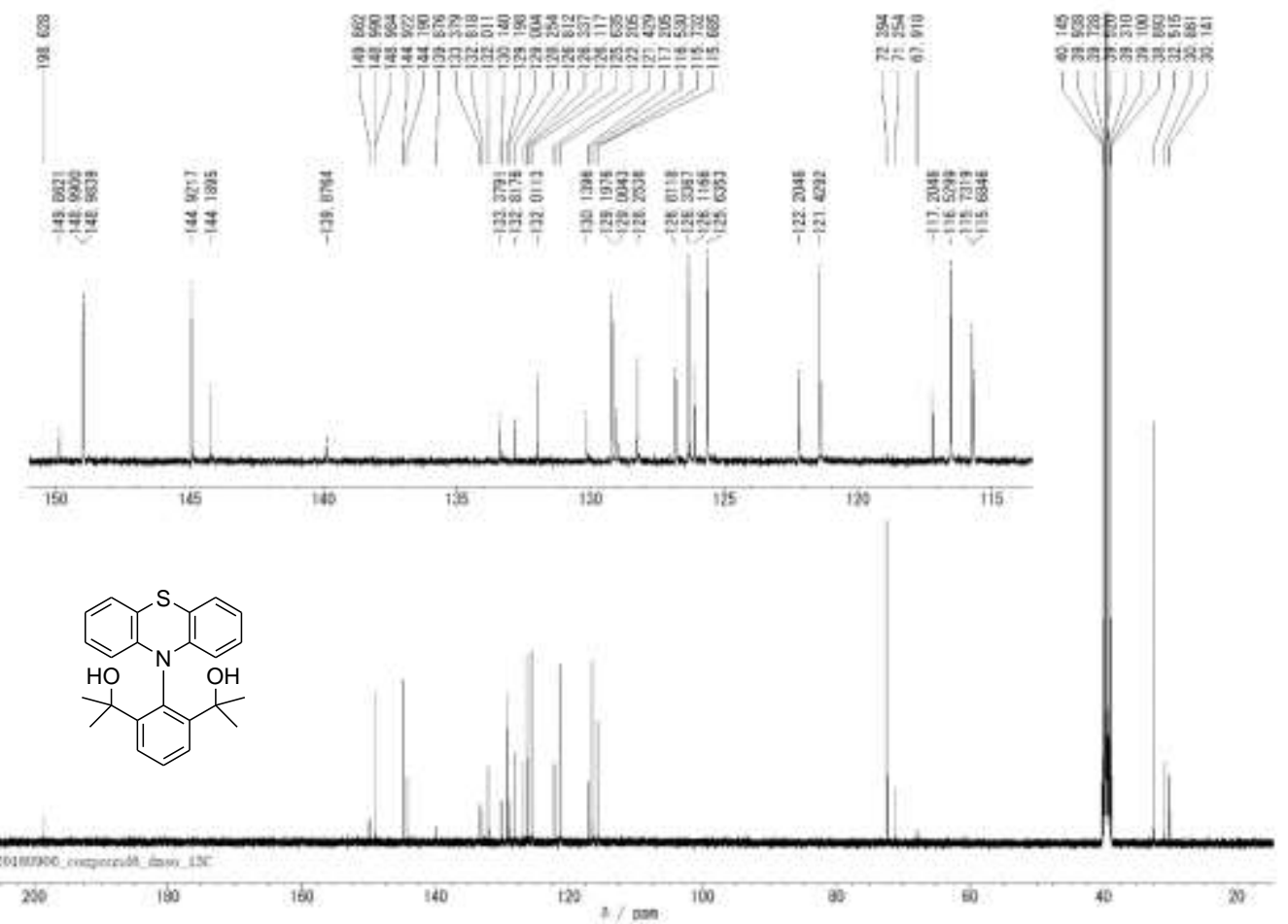

${ }^{13} \mathrm{C}$ NMR spectrum of 8 (DMSO- $\left.d_{6}, 100 \mathrm{MHz}\right)$. 


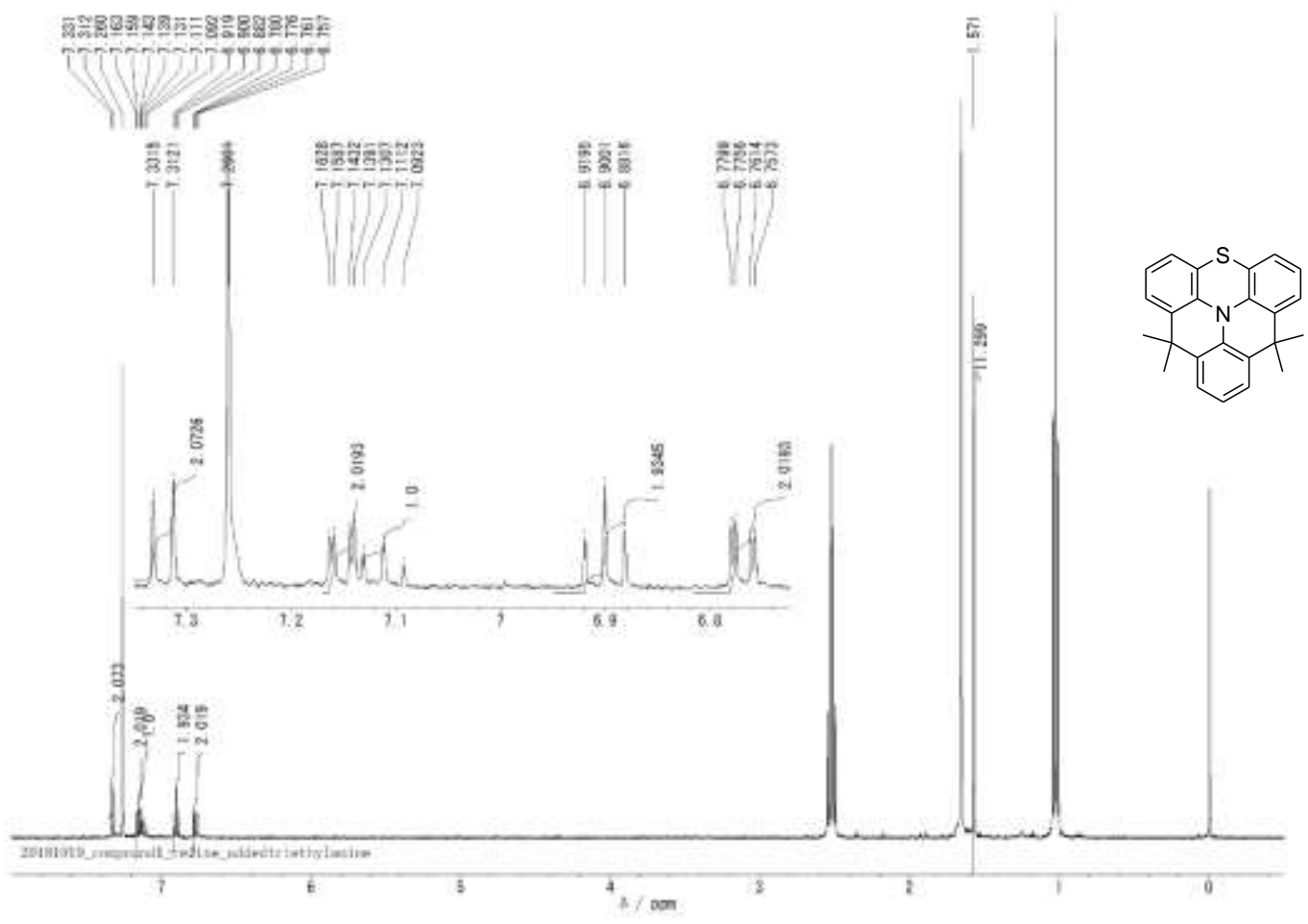

${ }^{1} \mathrm{H}$ NMR spectrum of $4\left(\mathrm{CDCl}_{3} / \mathrm{Et}_{3} \mathrm{~N}, 400 \mathrm{MHz}\right)$.

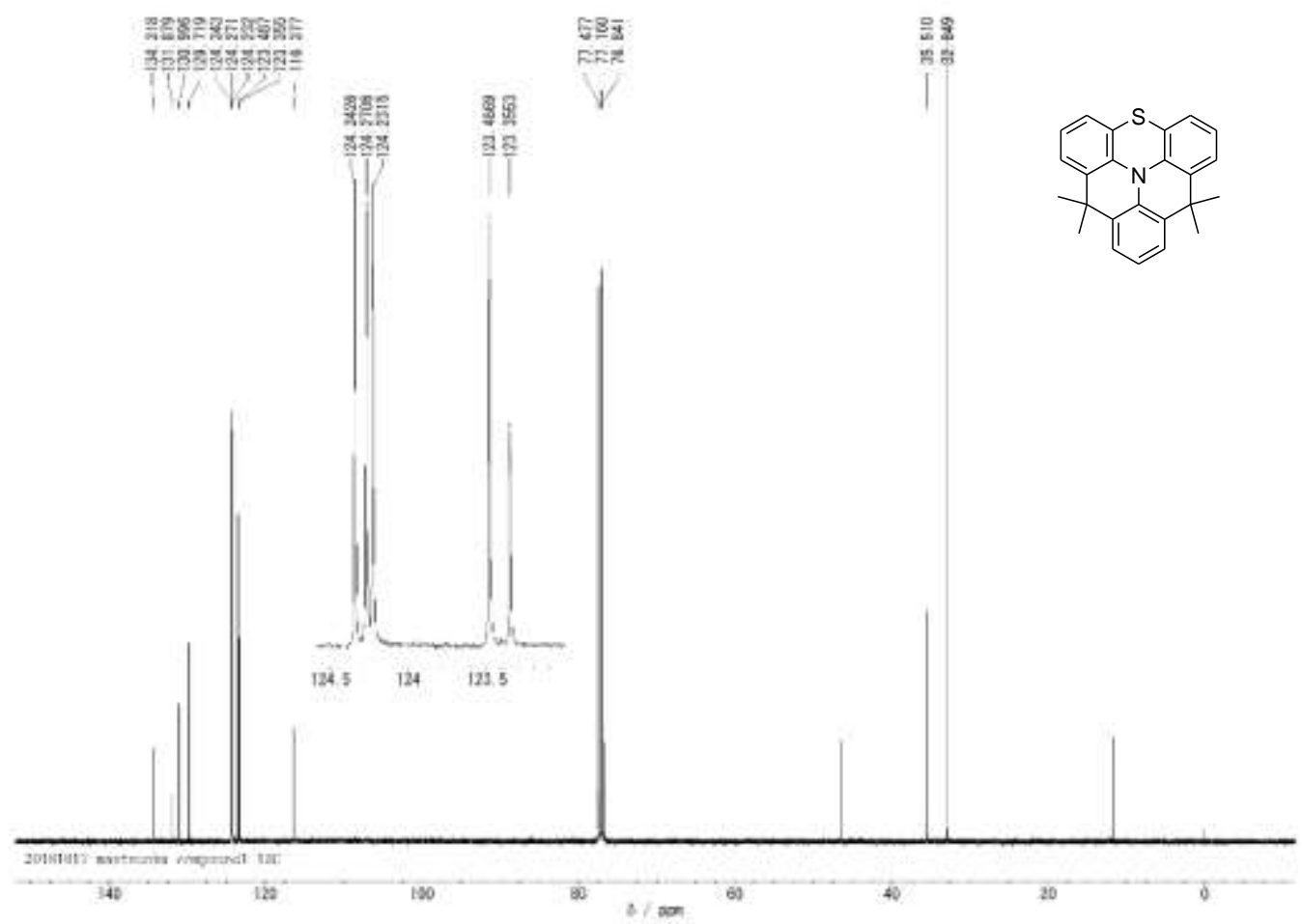

${ }^{13} \mathrm{C}$ NMR spectrum of $4\left(\mathrm{CDCl}_{3} / \mathrm{Et}_{3} \mathrm{~N}, 100 \mathrm{MHz}\right)$. 


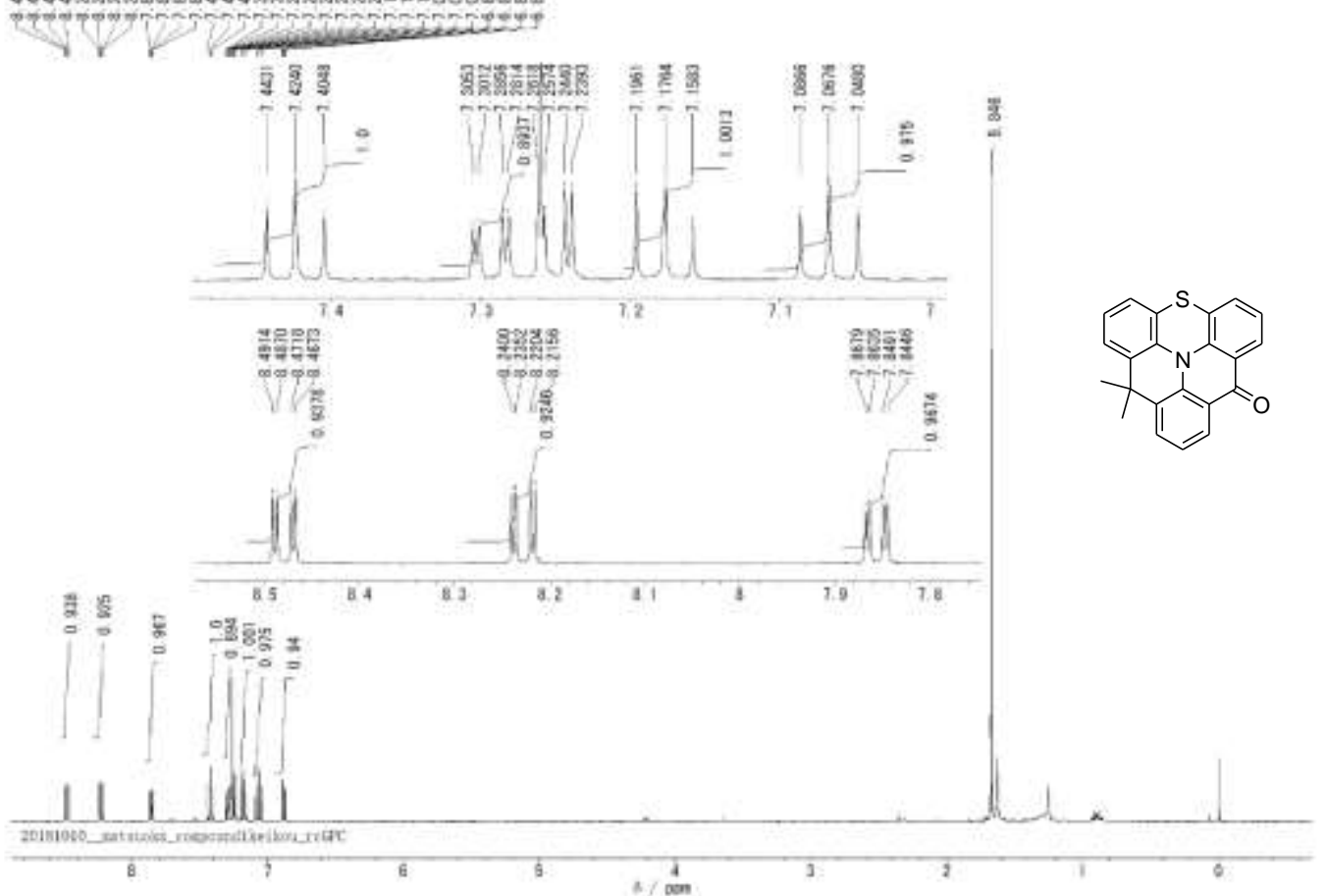

${ }^{1} \mathrm{H}$ NMR spectra of $5\left(\mathrm{CDCl}_{3}, 400 \mathrm{MHz}\right)$.

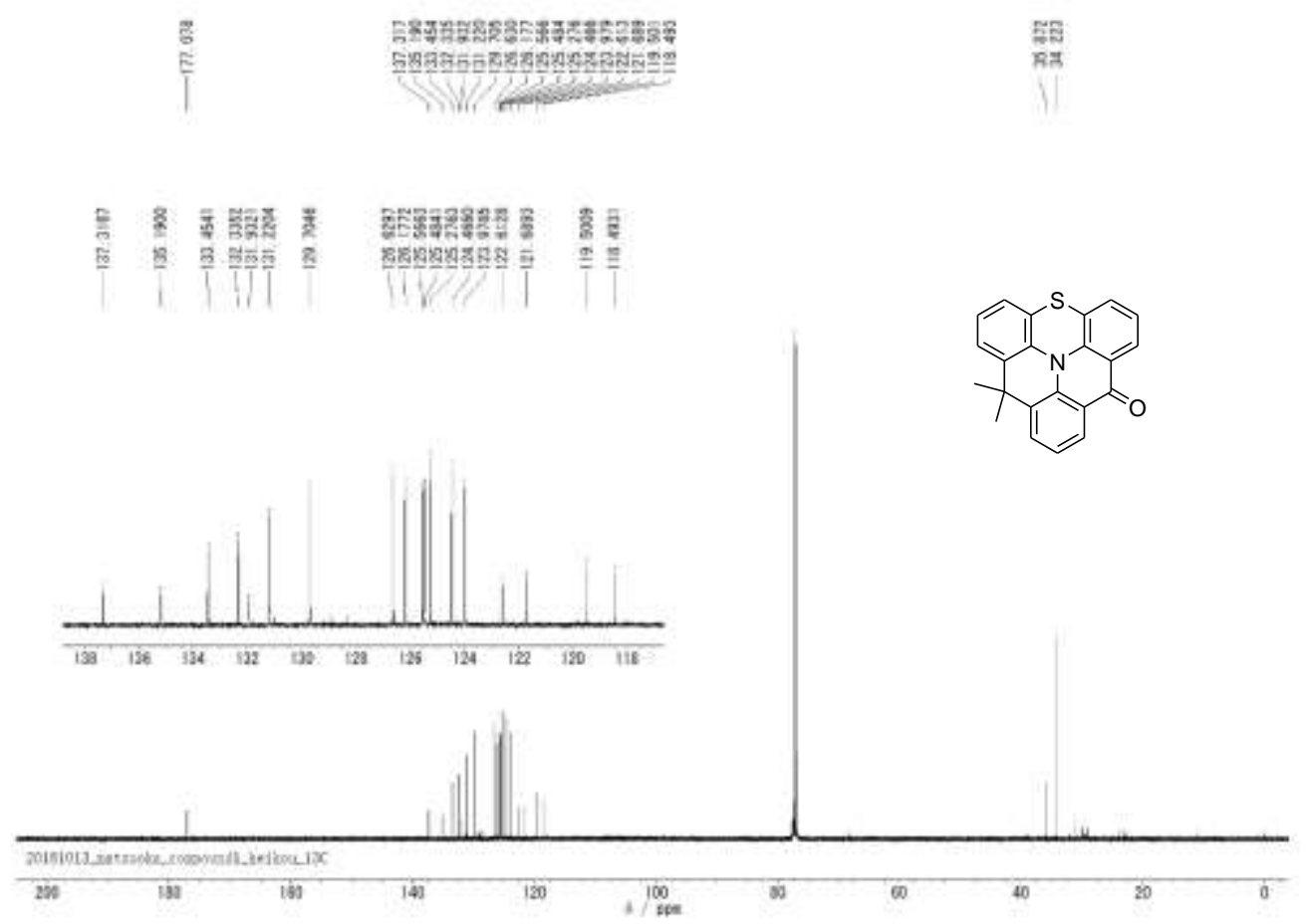

${ }^{13} \mathrm{C}$ NMR spectra of $5\left(\mathrm{CDCl}_{3}, 100 \mathrm{MHz}\right)$. 


\section{ESR spectral data}

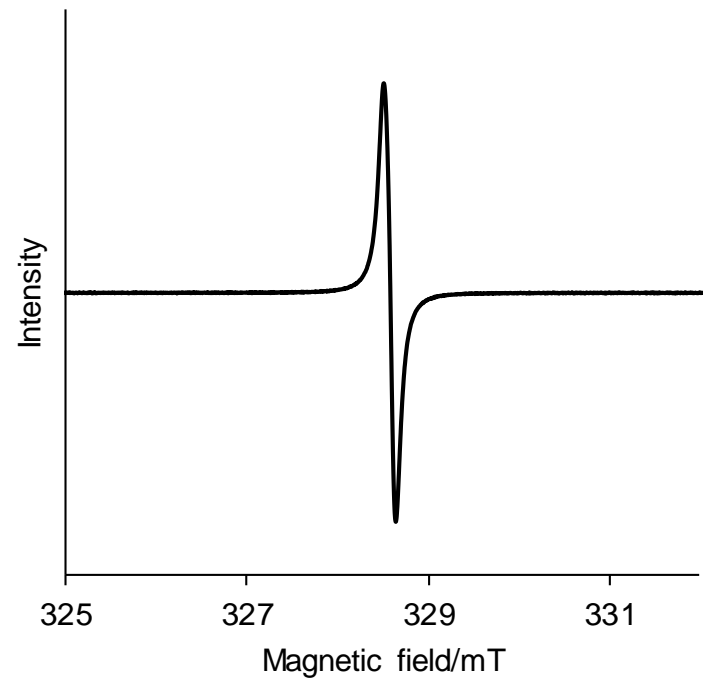

Figure S14. ESR spectrum of $\mathbf{4}^{++}$in the solid state. Even though an ESR signal was observed, DFT calculations using the Yamaguchi scheme clearly suggested antiferromagnetic exchange interactions in the dimeric structure (Table S15).

Table 15. Calculated Total Energies of Triplet and Singlet States, and Exchange Interactions $\left(\left(J_{\text {calc }} / k_{\mathrm{B}}\right) / \mathrm{K}\right)$ of Radical Cation Dimer of $\mathbf{4}^{++a}$

\begin{tabular}{cccc}
\hline Spin state & $E / \mathrm{au}$ & $\left\langle S^{2}\right\rangle$ & Exchange interaction $\left(J_{\text {cald }} / k_{\mathrm{B}}\right) / \mathrm{K}$ \\
\hline$S=1$ & -2759.341418 & 2.020063 & -816 \\
$S=0$ & -2759.3444647 & 0.840368 & \\
\hline
\end{tabular}

${ }^{a}$ The single-point calculations were performed using density functional theory at UB3LYP/6-31G(d,p) level of theory based on the X-ray crystallographic result. ${ }^{\mathrm{S} 1}{ }^{b}$ The total energy calculated using UHF-SCF procedure involving spin contamination due to the higher spin states. In order to estimate the exchange interaction, a compensation equation, $J_{\text {calc }}=\left({ }^{\mathrm{LS}} E-{ }^{\mathrm{HS}} E\right) /\left(\left\langle{ }^{\mathrm{HS}} S^{2}\right\rangle-\left\langle{ }^{\mathrm{LS}} S^{2}\right\rangle\right)$. 


\section{References}

1. Frisch, M. J.; Trucks, G. W.; Schlegel, H. B.; Scuseria, G. E.; Robb, M. A.; Cheeseman, J. R.; Scalmani, G.; Barone, V.; Mennucci, B.; Petersson, G. A.; Nakatsuji, H.; Caricato, M.; Li, X.; Hratchian, H. P.; Izmaylov, A. F.; Bloino, J.; Zheng, G.; Sonnenberg, J. L.; Hada, M.; Ehara, M.; Toyota, K.; Fukuda, R.; Hasegawa, J.; Ishida, M.; Nakajima, T.; Honda, Y.; Kitao, O.; Nakai, H.; Vreven, T.; Montgomery, J. A., Jr.; Peralta, J. E.; Ogliaro, F.; Bearpark, M.; Heyd, J. J.; Brothers, E.; Kudin, K. N.; Staroverov, V. N.; Kobayashi, R.; Normand, J.; Raghavachari, K.; Rendell, A.; Burant, J. C.; Iyengar, S. S.; Tomasi, J.; Cossi, M.; Rega, N.; Millam, J. M.; Klene, M.; Knox, J. E.; Cross, J. B.; Bakken, V.; Adamo, C.; Jaramillo, J.; Gomperts, R.; Stratmann, R. E.; Yazyev, O.; Austin, A. J.; Cammi, R.; Pomelli, C.; Ochterski, J. W.; Martin, R. L.; Morokuma, K.; Zakrzewski, V. G.; Voth, G. A.; Salvador, P.; Dannenberg, J. J.; Dapprich, S.; Daniels, A. D.; Farkas, Ö.; Foresman, J. B.; Ortiz, J. V.; Cioslowski, J.; Fox, D. J. Gaussian 09; Revision D.01; Gaussian Inc.: Wallingford CT, 2009.

2. Stoll, S.; Schweiger, A. EasySpin, a Comprehensive Software Package for Spectral Simulation and Analysis in EPR. J. Magn. Reson. 2006, 178, 42.

3. Zhdankin, V. V.; Koposov, A. Y.; Litvinov, D. N.; Ferguson, M. J.; McDonald, R.; Luu, T.; Tykwinski, R. R. Esters of 2-Iodoxybenzoic Acid: Hypervalent Iodine Oxidizing Reagents with a Pseudobenziodoxole Structure. J. Org. Chem. 2005, 70, 6484.

4. Dolomanov, O. V.; Bourhis, L. J.; Gildea, R. J.; Howard, J. A. K.; Puschmann, H. OLEX2: a Complete Structure Solution, Refinement and Analysis Program. J. Appl. Cryst. 2009, 42, 339.

5. Sheldrick, G. M. SHELXT-Integrated Space-Group and Crystal-Structure Determination. Acta Crystallogr., Sect. A 2015, 71, 3.

6. Sheldrick, G. M. Crystal Structure Refinement with SHELXL. Acta Crystallogr., Sect. C 2015, 71, 3. 